\title{
Varying Impedance "Orbital Impedance Stability" Graphene Based Supercapacitor Nanofiber Electrodes - Utilizing A New Direct Method of Studying Impedance Based on Actual Experimental Data
}

\author{
Mustafa H. Mustafa* \\ University of Illinois at Chicago, Engineering \& Research Facility, 842 West Taylor Street, Chicago, IL 60607-7022. \\ *Corresponding Author Email: mmusta4@gmail.com
}

\section{DOI: 10.2478/acmy-2019-0008}

Abstract:

Single cell supercapacitors with electrodes of varying amounts of graphene and carbon black, formed via the electrospinning process with a carbon-based Polyacrylonitrile (PAN), were tested in $1 \mathrm{M} \mathrm{H}_{2} \mathrm{SO}_{4}$. From the tested samples, the overall data indicates no correlation between impedance and capacitance energy values. However, the breakdown of the various samples showed mixed results of; good correlations between lower impedance resulting in higher and lower capacitance; while other samples showed higher impedance correlating to both higher and lower capacitance. No correlation was observed between the Impedance value and the thickness of the samples. Furthermore, carbon mole content was not a major factor in determining impedance; therefore, structure is not a major contributor to impedance. Whereas, carbon mole content is a major contributor to capacitance energy; Hence, impedance provides an alternative control point to increasing energy ( 2-10X times), that can be retrofitted to existing systems, or to increase the energy storage beyond current levels by adjusting/controlling impedance in new designs. The data is indicating impedance is not constant and is varying. The mechanism of varying impedance is unclear and requires further research. However, it is thought to mimic the energy level and stability of matter (atoms). Therefore, impedance varies or oscillates accordingly to achieve an impedance level stability, and hence the term "Orbital Impedance Stability". Thoughts into Impedance being an Energy Field, to be provided in next publication (In-sha'-ALLAH). This research is concluding that our conventional understanding of impedance is limited in scope. New approaches and further research is needed to better understand impedance behavior. A better understanding of impedance is essential to a breakthrough in energy storage devices from capacitors and batteries, to electric generation and distribution of energy, to magnetic levitation, medical drugs and other energy improvements.

Keywords: Impedance; Orbital Impedance Level, New Method or Direct Method of Studying Impedance; Supercapacitor; Nanofiber; Electrode; Graphene; Electrospinning

\subsection{Introduction}

This is a continuation of graphene based supercapcitance research [1]. Carbon-based supercapacitors offer high surface area, non-toxicity, chemical stability, abundance and low cost which are ideal energy storage devices [2]. There are several methods to manufacture supercapacitors, screen printing, coating and spray technology [3-5]. Some of these methods require a binder, like polytetrafluoroethylene (PTFE), to compact carbon particles for capacitance storage. Furthermore, many future applications are ideal candidates such as hybrids systems, biosensing and flexible supercapacitors [6-8]. Electrospinning is a technique to produce polymer nanofibers $[9,10]$. Carbon nanofibers mats have previously been used as electrodes for energy storage $[11,12]$. However, Research continues on electrospinning to better understand the stagnation plan, jet path and electric field strength, near field and TipInduced effects $[9,13]$.

Research on electrospun activated carbon nanofibers points to strong dependency of charge resistance and surface area on supercapacitors [9]. Other research has shown that pore size and surface area distribution are improvements but not a major factor in increasing capacitance [14,15]. However, anomalous increase in carbon capacitance for pore sizes less than $1 \mathrm{~nm}$, using carbide-derived carbons (CDCs), has been reported [16]. Furthermore, Largeot et al. [17] reported on the relationship between ion size and pore size. Also, Ania et al. [18] reported on the polarization induced ion distortion in the pores of carbon. And finally, a pseudo-faradaic charge transfer type behavior has been reported in the porous regions by Frackowiak et al. [19]. This research will investigate the impedance of supercapacitors created from various graphene and carbon black nanofiber mats formed by the electrospinning process. Carbon activation by physical or chemical means would not be used to reduce variability amongst tested samples. The Nanofiber material will be characterized by an energy dispersive X-ray (EDX) spectroscopy, which determines the emissions spectrum of the sample, by measuring the emitted peak energy; and the carbon structure would be analyzed with a transmission electron microscope (TEM).
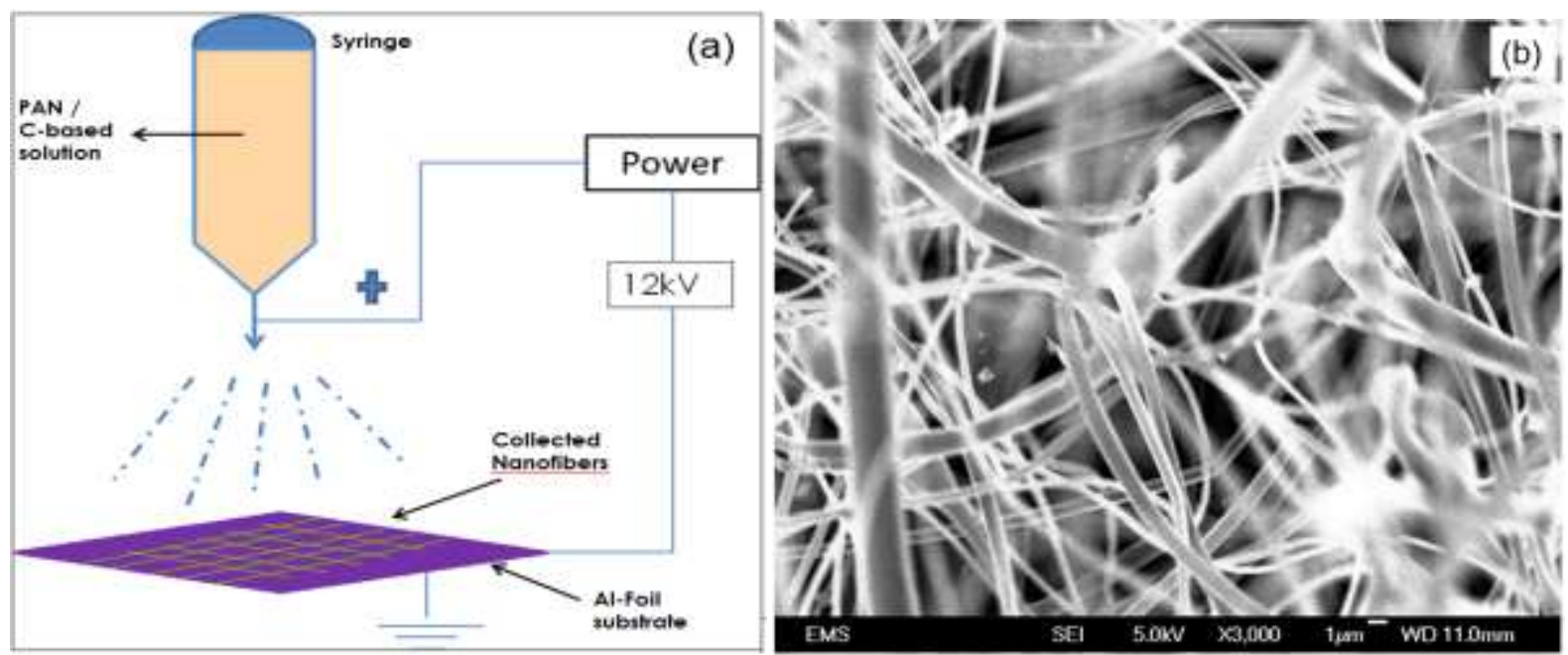

Figure 1: a) Electrospinning process illustration, b) Scanning Electron Microscope (SEM) of collected nanofiber mats

\subsection{Experimental}

\subsection{Sample Preparation}


Carbon nanofiber electrode samples were prepared using the electrospinning process with solution of 8\% by wt. PAN-Polyacrylonitrile (MW 150,000) in $100 \%$ DMF-N-N dimethylformamide (solvent) and with a 15\%, 25\% and 35\% by weight of graphene oxide ( N006-010-P) from Angstron Materials added to the $8 \%$ by wt. PAN. Additional samples were made by adding $10 \%$ by wt. carbon black (CB) from Cabot to the $8 \%$ by wt. PAN mixture with the same graphene concentrations. The electrospinning process was used to create the nanofiber mat electrodes. A brief description of the process is given below; additional information on the process and techniques has been defined in the literature $[8,20]$.

The PAN, DMF and additional carbon sources (graphene oxide, carbon black) were placed in a $20 \mathrm{ml}$ standard glass container and placed on a heated rotator for 12 hours to stir the mixture and create a homogenous solution. The solution was loaded into a 10cc 18GA needle syringe and allowed to flow at a rate of approximately $1.0 \mathrm{~mL} / \mathrm{hr}$ at a voltage potential of $12.5 \mathrm{kV}$ and at a separation distance of $16 \mathrm{~cm}$ from the aluminum foil collector. The nanofibers were collected on the aluminum foil, creating the nanofiber mats. The samples were then heat treated to $800^{\circ} \mathrm{C}$ by first ramping up the temperature at a rate of $5^{\circ} \mathrm{C} / \mathrm{min}$ from room temperature to $350^{\circ} \mathrm{C}$. A flow of nitrogen at $5 \mathrm{ccm}$ was then introduced and the samples were heated from $350^{\circ} \mathrm{C}$ to $800^{\circ} \mathrm{C}$ at a rate of $7^{\circ} \mathrm{C} / \mathrm{min}$. The samples were then cooled back down to room temperature. The heat treatment of the nanofiber mats carbonizes the (PAN) at elevated temperatures with an inert atmosphere, which removes non-carbon elements. Carbon activation refers to the process of forming pores and pockets in carbon to increase its surface area. Carbon activation is done by physical means such as a suitable oxidizing gas agent $\left(\mathrm{O}_{2}, \mathrm{CO}_{2}\right.$ or steam), or by chemical activation such as $\left(\mathrm{KOH}, \mathrm{H}_{3} \mathrm{PO}_{4}, \mathrm{ZnCl}_{2}\right)$, followed by carbonization at $400^{\circ}$ to $900^{\circ} \mathrm{C}$ [21]. Carbon activation was not performed on the samples to reduce variability and focus on the graphene and carbon black effects on supercapcitance.

Two-electrode supercapacitor test cells were assembled by using two nanofiber mats and separated by an insulator (Cellgard membrane 3501) as shown in Figure 2. The opposite ends of the nanofiber mat samples were in contact with a nickel wire mesh used as a current collector. The two-electrode assembly was clamped with a spring loaded plastic clip (providing an analogy of bolt stretch, used in a conventional supercapacitor). A cotton pad was placed on each end of the nickel wire mesh and wetted with $1 \mathrm{M} \mathrm{H}_{2} \mathrm{SO}_{4}$. The average area of the nanofiber mat was approximately $0.35 \mathrm{~cm}^{2}$ and the depth varied from 0.15-1.10mm thickness. A total of five (5) test cells for each electrode composition were constructed.

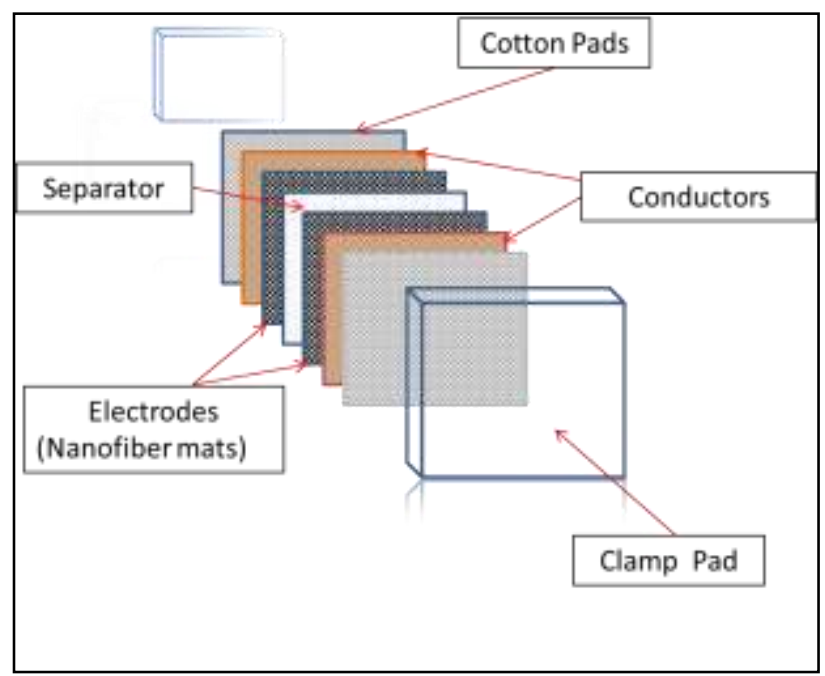

Figure 2: Single-cell Two-electrode assembly with nanofiber mats

\subsection{Cyclic Voltammetry (CV)}

Supercapacitor test cell performance was measured electrochemically by cyclic voltammetry (CV). The two-electrode test cell was subjected to a voltage range of $0-0.9 \mathrm{~V}$ and six scan rates between $10-500 \mathrm{mV} / \mathrm{s}$. The scans were performed using a Princeton Applied Research PARSTAT 2273 Advanced Electrochemical System (Potentiostat/Galvanostat) controlled with Power Suite software. In order to perform the scans as a two-electrode system, the counter and reference wires of the instrument were connected to one side of the supercapacitor nanofiber electrode assembly, while the working electrode connection was connected to the other electrode. The linear portion of the (CV) discharge curve, in the range of 0.45 to $0.54 \mathrm{~V}$, was used to calculate the specific capacitance at a scan rate of $10 \mathrm{mV} / \mathrm{s}$, further information on capacitance definition can be found [1]

The capacitance is defined as:

$\mathrm{C}=\mathrm{Q} / \mathrm{V}$

where $\mathrm{C}$ is the capacitance in Farads (F), $\mathrm{Q}$ is the charge in coulombs (i.e., the area under the (CV) curve), and V is the voltage (V).

The current I , can also be defined as:

$I=(d Q / d t)$

where I is the current in amperes, [ $\mathrm{dQ}$ is charge in coulombs and dt is the discharge time (s) ] taken from a linear slope, in the range of $0.45-0.54 \mathrm{~V}$.

Therefore, the capacitance becomes:

$C=d Q / d V$

where $\mathrm{dV}$ is the potential variation in the time range $\mathrm{dt}$

The specific capacitance $(\mathrm{F} / \mathrm{g})$ is calculated by:

$\mathrm{C}_{\mathrm{m}}=\mathrm{C} / \mathrm{m}$

where $C_{m}$ is the specific capacitance in Farads per gram of the sample $(F / g)$ and $m$ is the

weight in grams $(\mathrm{g})$ 


\subsubsection{Electrochemical Impedance Spectroscopy (EIS) Measurements}

The electrochemical impedance (EIS) of the samples was measured using Gamry Instruments PARSTAT 2273 potentiostat/galvanostat and Gamry Framework Software. The imposed ac voltage was set to $10 \mathrm{mV}$ and the frequency range $(10 \mathrm{mHz}-100 \mathrm{kHz})$

The Electrical impedance is calculated as follow:

$\mathrm{Z}=\mathrm{Z}(\mathrm{re})+\mathrm{i}(\mathrm{im})$

where $\mathrm{Z}$ is Electrochemical Impedance, Z(re) is Real part of Impedance, i Z(im ) is Imaginary part of Impedance [22].

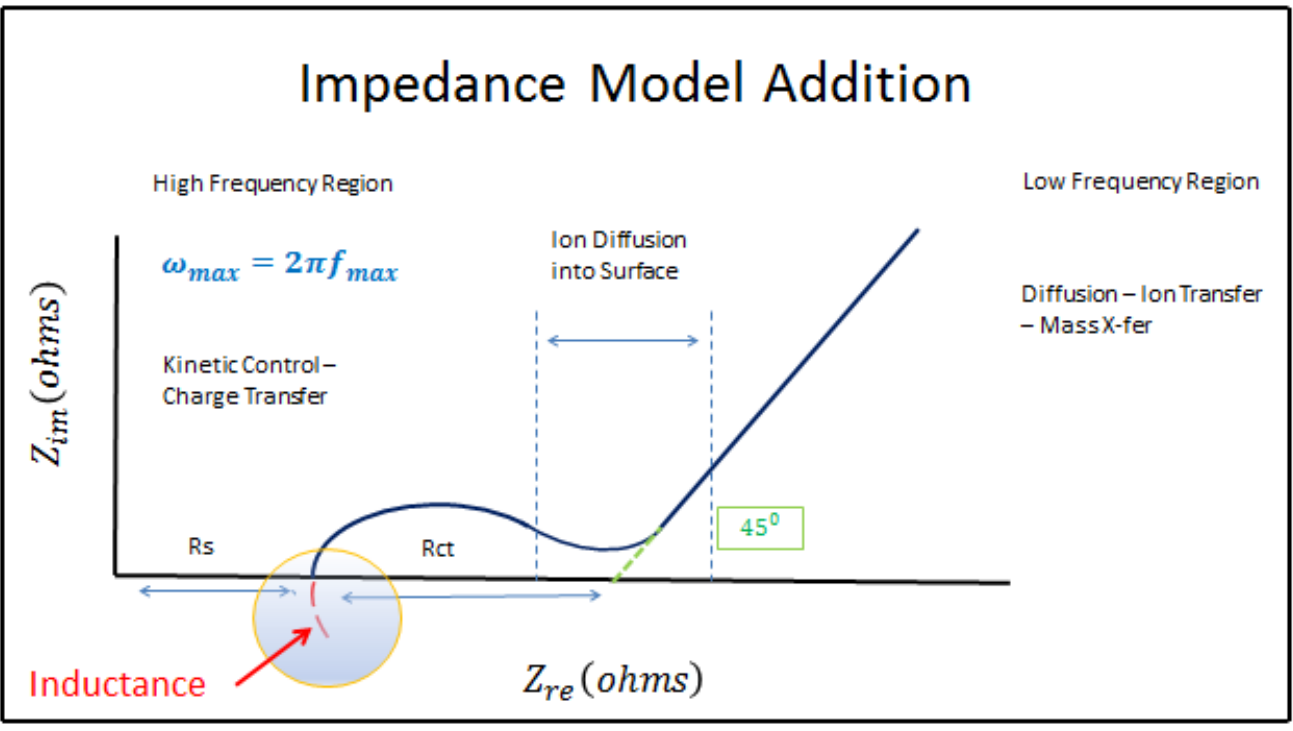

Figure 3: Modified De Levie Impedance Model with Inductance

The Impedance of the nanofiber supercapacitors was initially analyzed using circuit model analysis. A basic form of an Inductor, Resistor and Capacitor circuit in series can be described in the following equation:

$V \cos (w t)=L(d I a / d t)+R I a+(1 / C) \int I a(t) d t$

V-Voltage(V), L-Inductor ( H), R-Resistance (ohms) , C-Capacitance ( Farads), Ia - current (amps), t- time (s), w- angle (rad)

Assuming the solution in the form

$V \cos (w t)=V e^{(i w t)}$

Let the solution of ( Ia ) be a complex number ( I ),

$I a=I e^{(i w t)}$

Therefore equation ( 5.2 ) becomes:

$V e^{(i w t)}=[(i w L)+R+(1 /(i w C))] I^{*} e^{(i w t)}$

Which reduces to:

$V=I^{*}[(i w L)+R+(1 /(i w C))]$

This is in the form $\left(\mathrm{V}=\mathrm{I}^{*} \mathrm{Z}\right)$, where $\mathrm{Z}$ is complex impedance and is equal to:

$Z=[R+(i w L)+(1 /(i w C))]$

\subsection{Spectrum and Image Analysis}

A Hitachi Ltd ( S-3000N), Energy Dispersive X-ray ( EDX ) spectroscopy from Oxford was employed to measure chemical composition of the nanofiber samples. Image analysis of carbon nanofibers were performed with a JEOL JEM-3010 with Gatan digital cameras and Thermo-Noran XEDS transmission electron microscope (TEM).

\subsection{Results and Discussion}

\subsection{Cyclic Voltammetry (CV) Results}

Figure 4 and Figure 5 show representative (CV) scans for the supercapacitor test cells containing nanofiber electrodes with 8\% PAN, 15\%, 25\% and 35\% graphene and with/without $10 \%$ carbon black. A sample with only 8\% PAN was also tested as shown in Figure 5(d). A total of 5 separate tests were performed for each electrode; the capacitance vs impedance values are shown in Figure 9 and 10. 

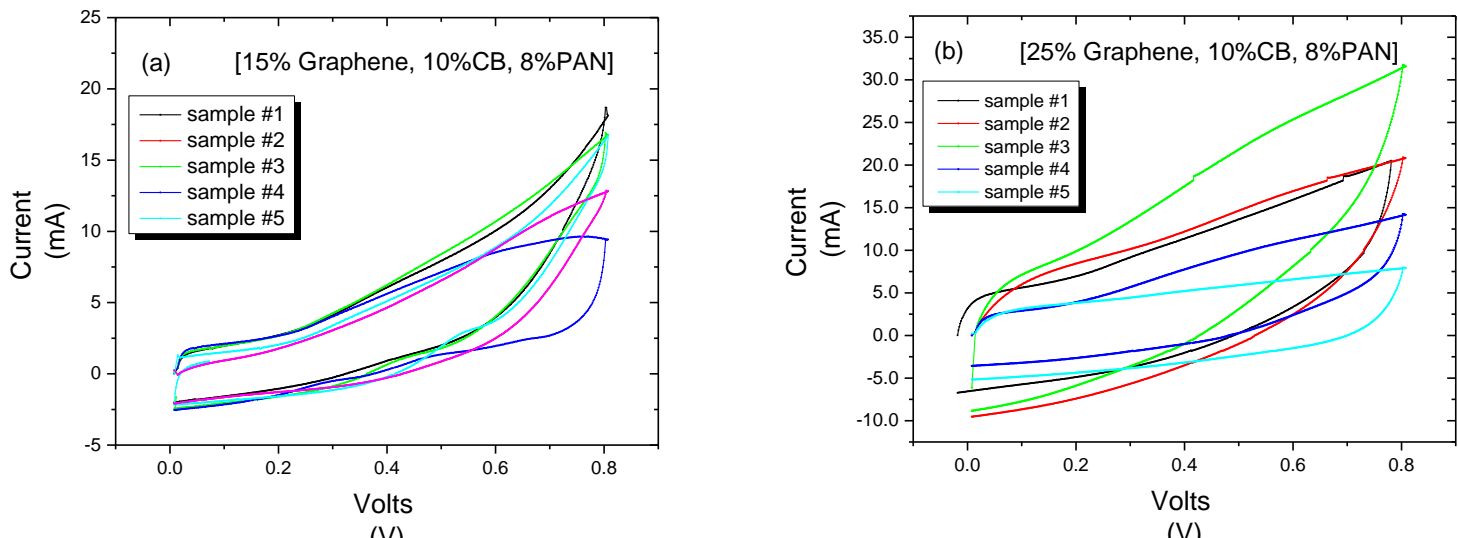

(V)

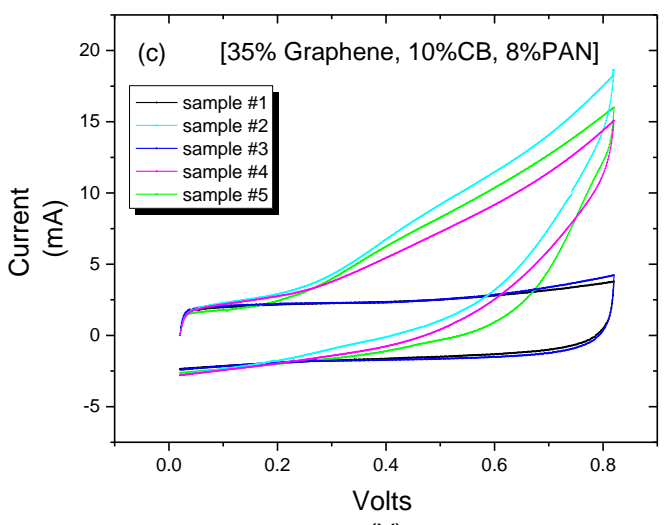

(V)

(V)

Figure 4: (a) CV graphs for $15 \%$ graphene, $10 \% \mathrm{CB}, 8 \% \mathrm{PAN}$, in $1 \mathrm{M} \mathrm{H}_{2} \mathrm{SO}_{4}$; (b) CV graphs for 25\% graphene, $10 \% \mathrm{CB}, 8 \% \mathrm{PAN}$, in $1 \mathrm{M} \mathrm{H}_{2} \mathrm{SO}{ }_{4}$; (c) $\mathrm{CV}$ graphs for $35 \%$ graphene, $10 \% \mathrm{CB}, 8 \% \mathrm{PAN}$, in $1 \mathrm{M} \mathrm{H}_{2} \mathrm{SO}_{4}$

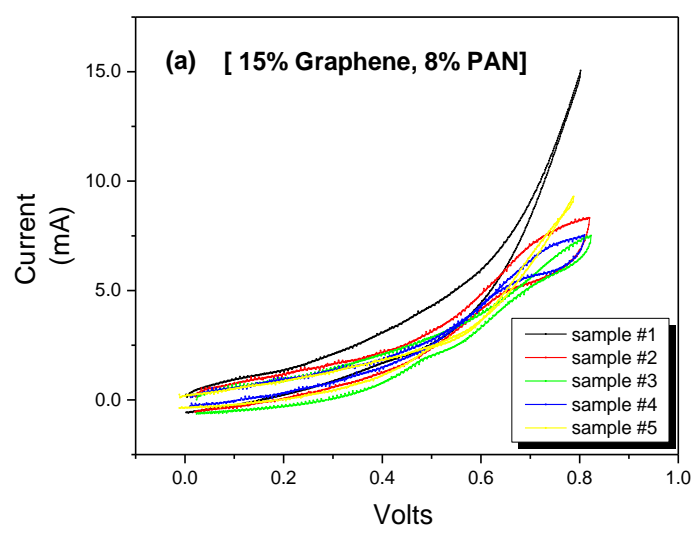

(V)

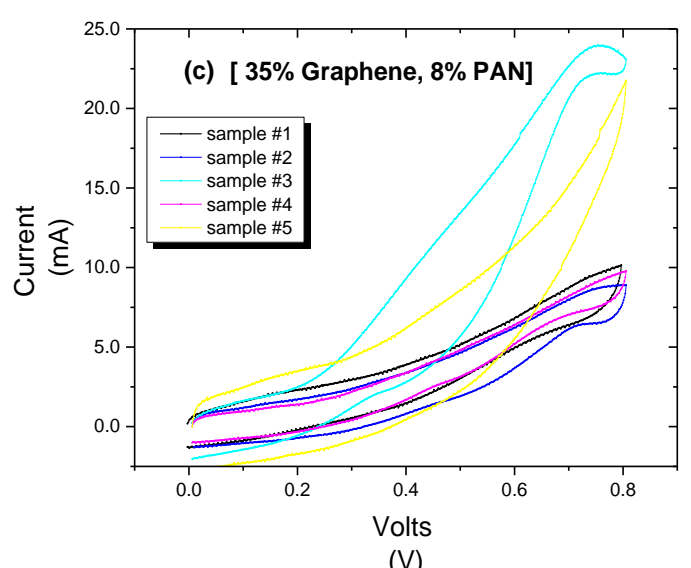

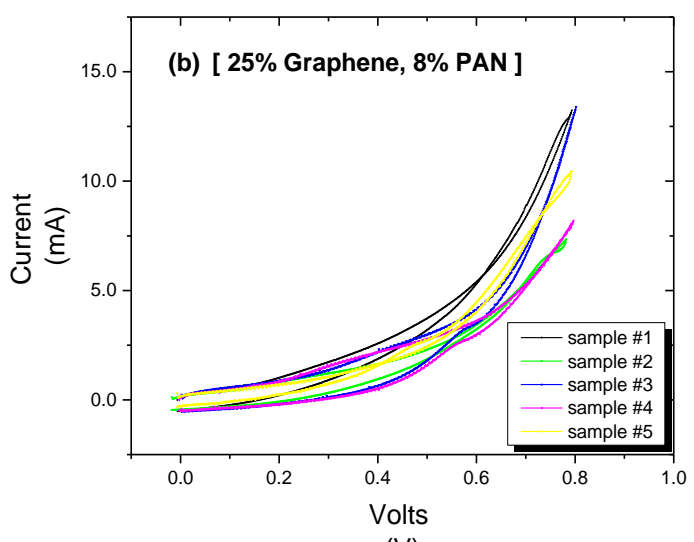

(V)

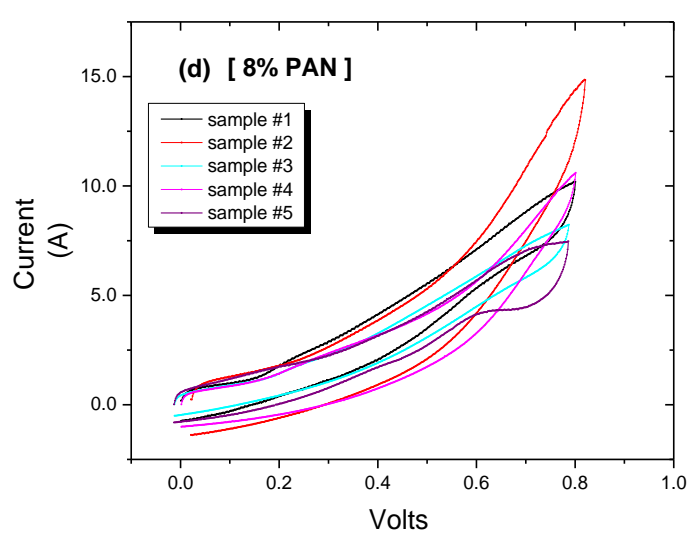

(V)

Figure 5: (a) CV graphs for 15\% graphene, 8\% PAN, in $1 \mathrm{M} \mathrm{H}_{2} \mathrm{SO}_{4}$; (b) $\mathrm{CV}$ graphs for 25\% graphene, $8 \% \mathrm{PAN}$ in $1 \mathrm{M} \mathrm{H}_{2} \mathrm{SO}{ }_{4}$; (c) $\mathrm{CV}$ graphs for 35\% graphene, $8 \%$ PAN, in $1 \mathrm{M} \mathrm{H}_{2} \mathrm{SO}_{4}$; (d) $\mathrm{CV}$ graphs for $8 \%$ PAN only, in $1 \mathrm{M} \mathrm{H}_{2} \mathrm{SO}_{4}$. 


\subsection{Electrochemical Impedance Spectroscopy (EIS) and Circuit Model Analysis (CMA) Results}

The (EIS) plots were used to qualitatively compare the impedance values of the different samples. The electrical Impedance of the samples was measured by using Gamry Framework Instrumentation and the circuit model analysis was done using Gamry Echem Analyst software. The circuit model analysis and curve fit are shown in Figure 8. An electrochemical capacitor has both ionic and electronic resistance per traditional understanding. The ionic resistance is due to separator resistance and ion conduction in the electrode pores ( ion movement within the electrodes ). The electronic resistance is mainly due to wire resistance and the bulk resistance of electrode material such as particle to particle contact. The (EIS) plots (Nyquist plot) were used to estimate the capacitance of the electrode by measuring the impedance value of the high frequency Figure 3. Figure 6 and Figure 7 show the (EIS) Plots of the impedance for the supercapacitor test cells containing nanofiber electrodes with $8 \%$ PAN, 15\%, 25\% and 35\% graphene and with/without $10 \%$ carbon black. A sample with only 8\% PAN was also tested as shown in Figure 7 (d). A minimum of 5 separate tests were performed for each electrode. (EIS) plots were initially used to estimate the capacitance of the samples by using Circuit Model Analysis (CMA), as a curve fit replication of tested samples Figure 8. The capacitance results of the (CMA) (from EIS data) did not correlate to the measured capacitance value from the (CV) data. In addition, correlating (EIS) impedance test data and (CV) test data using traditional methods was not feasible. Therefore, to correlate the (EIS) Impedance test data with the (CV) capacitance test data, a new guide was developed to bridge/combine the data of the two tests for analyzing impedance and capacitance. The new method combines the results of the (CV) and (EIS) test data.

The New Direct Approach to Studying Impedance:

The new method of studying impedance is based on the actual experimental data. Without assumptions or curve fit analysis from (EIS) (CMA), this approach readily reflects the actual samples tested.

The key to this method is to encompass the capacitor's discharge time within a full cycle (Frequency) in (EIS)

The (CV)/ (EIS) Bridging Formula:

$\operatorname{Dt}(\mathrm{CV}) \leftrightarrow \mathrm{Hz}$ ( EIS )

Dt (discharge time of capacitor in sec)

$\mathrm{Hz}$ (EIS) is the Frequency that encompasses Dt.

The simplest form of the new impedance method is to compare the capacitance of the (CV) test with the (EIS) impedance value at a specified frequency, for all samples.

To further increase accuracy of the method (Match the Power Output of the Capacitor with the Power output of the (EIS) test, however, in this research an estimate was made by:

Set (CV) voltage $\leftrightarrow$ (EIS) output voltage

Example: $10 \mathrm{mv} / \mathrm{s}$ scan rate (CV) $\leftrightarrow 10 \mathrm{mVac}$ (EIS)

Determine capacitor discharge time $\leftrightarrow$ within one full (EIS) cycle

Example: $10 \mathrm{~ms}$ discharge time $\leftrightarrow 100 \mathrm{~Hz}$ (Practical Capacitance Discharge time)

For more guidance on selecting the frequency \& Voltage, do the following steps.

1. Determine the voltage magnitude of the (CV) scan rate,

The scan rate used in this research is $(10 \mathrm{mV} / \mathrm{s})$, therefore, the magnitude is $10 \mathrm{mV}$.

2. Set the (EIS) output voltage magnitude to match the (CV) voltage magnitude.

Therefore, the output of the (EIS) test is (10mVac).

3. Determine the discharge time ( $\mathrm{t}$ ) of the capacitor being tested. ( or $\mathrm{t}=\mathrm{R}^{*} \mathrm{C}$ for estimate)

In this research, an arbitrary discharge time of $10 \mathrm{~ms}$ was used (most common).

4. Determine the frequency value that best matches the discharge time of the capacitor. In other words, what is the frequency that can cycle within the discharge time?

Therefore, at $10 \mathrm{~ms}$ discharge time, the frequency is $100 \mathrm{~Hz}$

At $100 \mathrm{~ms}$ discharge time, the frequency is $1 \mathrm{kHz}$.

5. Analyze the real measured impedance values (EIS) vs. capacitance (CV), at the specified frequency(s) that is equivalent to the discharge time of the tested sample,

The (10ms) discharge time corresponds to a frequency of $(100 \mathrm{~Hz})$ in (EIS).

6. Repeat the steps above with impedance values at different frequencies and scan rates as needed, or expand analysis around designated values.

7. For further future improvements, match the Capacitor's Power ( $\left.\mathrm{P}_{\mathrm{cap}}\right)$ from (CV), to be similar to the (EIS) Power $\left(\mathrm{P}_{\mathrm{EIS}}\right)$ in the impedance test. [ $\left.\mathrm{P}_{\mathrm{cap}}=\mathrm{P}_{\mathrm{EIS}}\right]$.

In summary,

- Match the voltage magnitude of the (CV) scan rate and (EIS) output voltage.

- Match the (EIS) frequency with the discharge time of the capacitor or tested sample.

- Initiate impedance vs. capacitance analysis around these conditions and expand as needed.

Ideally, it's best to match the Capacitor's Power ( $\mathrm{P}_{\text {cap }}$ ) from (CV), to the (EIS) Power (PeIs) in the Impedance test. [ $\mathrm{P}_{\text {cap }}=\mathrm{P}_{\text {eIs }}$ ].

The Impedance vs. Capacitance Results are shown in Figure 9 for the $100 \mathrm{~Hz}$ and Figure 11 for the $1 \mathrm{kHz}$ Frequencies. 


\subsection{Electrochemical Impedance Spectroscopy Results}

(8\% PAN, 10\% Carbon Black \& 15\%,25\%, 35\% graphene)
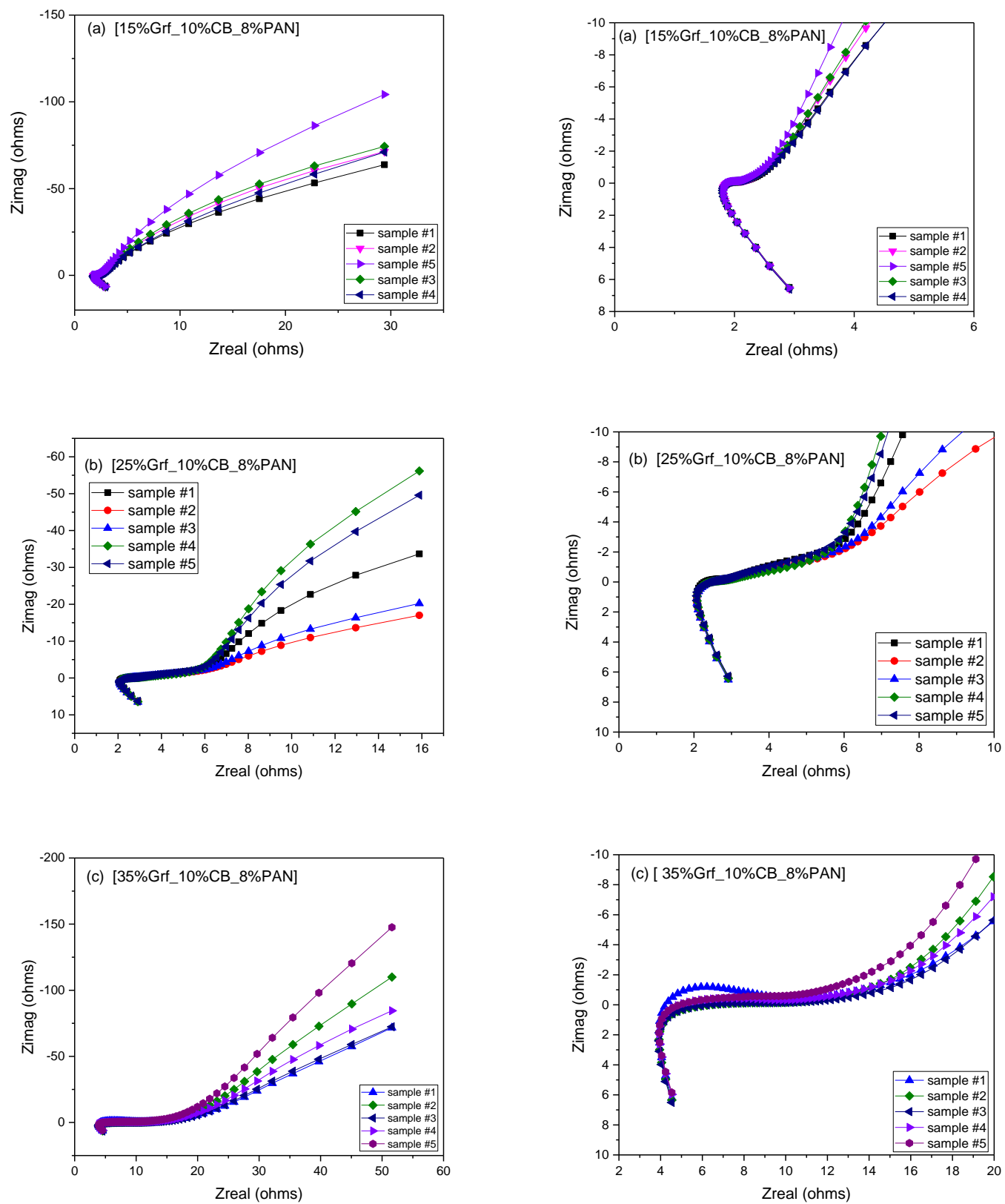

Figure 6: (a) Impedance graphs for $15 \%$ graphene, $10 \% \mathrm{CB}, 8 \% \mathrm{PAN}$, in $1 \mathrm{M} \mathrm{H}_{2} \mathrm{SO}_{4}$; (b) Impedance graphs for $25 \%$ graphene, $10 \% \mathrm{CB}, 8 \% \mathrm{PAN}$, in $1 \mathrm{M} \mathrm{H}_{2} \mathrm{SO} \mathrm{H}_{4}$ (c) Impedance graphs for $35 \%$ graphene, $10 \% \mathrm{CB}, 8 \% \mathrm{PAN}$, in $1 \mathrm{M} \mathrm{H}_{2} \mathrm{SO}_{4}$
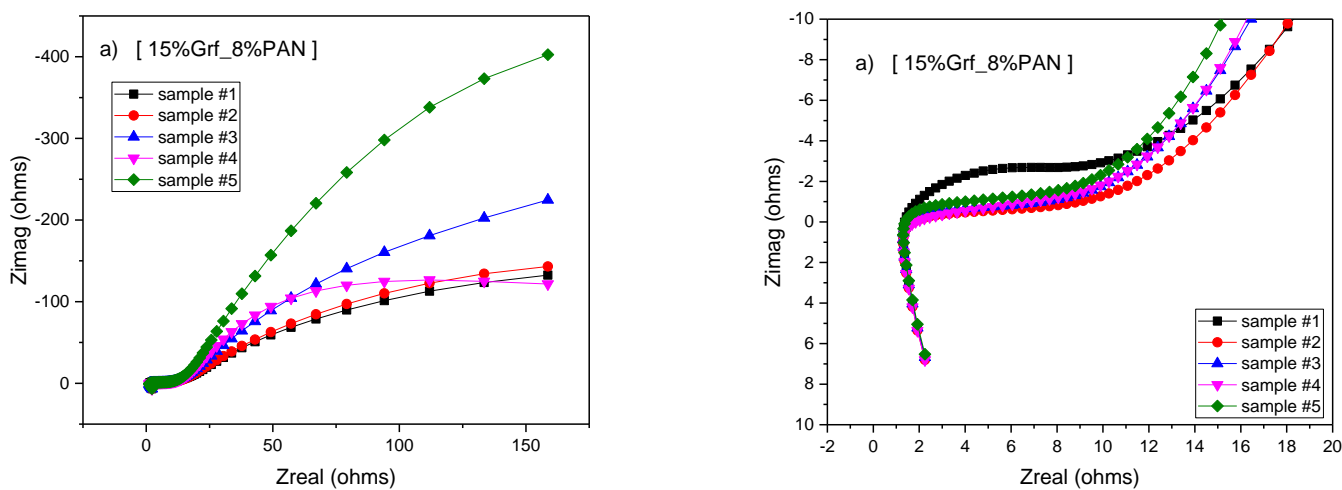

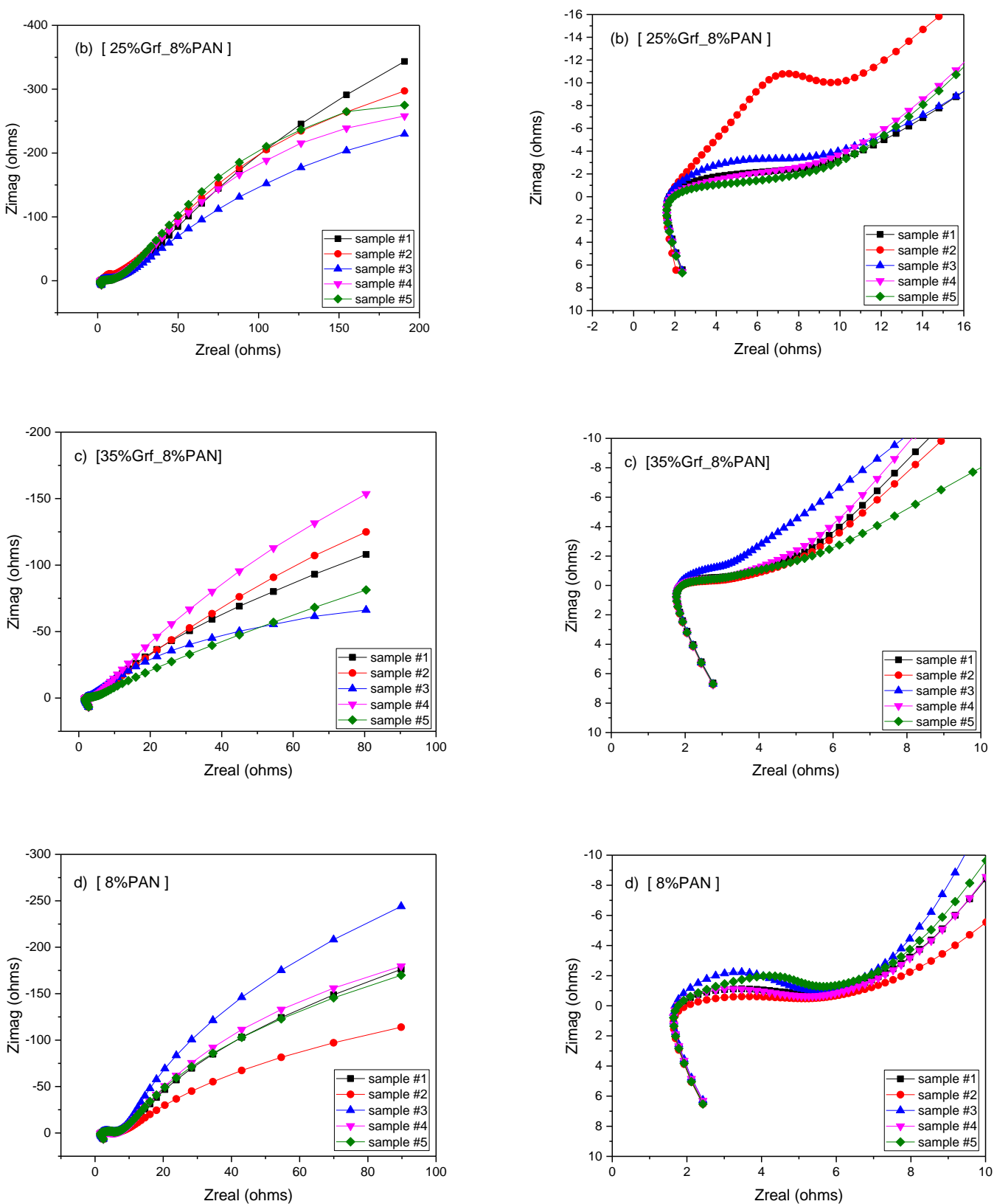

Figure 7: Impedance graphs for 15\%, 25\% \& 35\% graphene and 8\% PAN samples in 1M H2SO4; (a) Impedance graphs for 15\% graphene, 8\% PAN, in 1M $\mathrm{H}_{2} \mathrm{SO}_{4}$; (b) Impedance graphs for $25 \%$ graphene, $8 \% \mathrm{PAN}$, in $1 \mathrm{M} \mathrm{H}_{2} \mathrm{SO}_{4}$; (c) Impedance graphs for 35\% graphene, $8 \%$ PAN, in $1 \mathrm{M} \mathrm{H}_{2} \mathrm{SO}_{4}$; (d) Impedance graphs for $8 \%$ PAN in $1 \mathrm{M} \mathrm{H}_{2} \mathrm{SO}_{4}$

The electrochemical impedance (EIS) of the samples was measured using Gamry Instruments PARSTAT 2273 potentiostat/galvanostat and Gamry Framework Software. The imposed ac voltage was set to $10 \mathrm{mV}$ and the frequency range $(10 \mathrm{mHz}-100 \mathrm{kHz})$. The qualitative comparison of the impedance was done at the $100 \mathrm{~Hz}$ and $1 \mathrm{kHz}$ frequency range. The results of the impedance and capacitance are shown in Figures 9 for the $100 \mathrm{~Hz}$ Frequency and Figures 11 for the $1 \mathrm{kHz}$ Frequency.
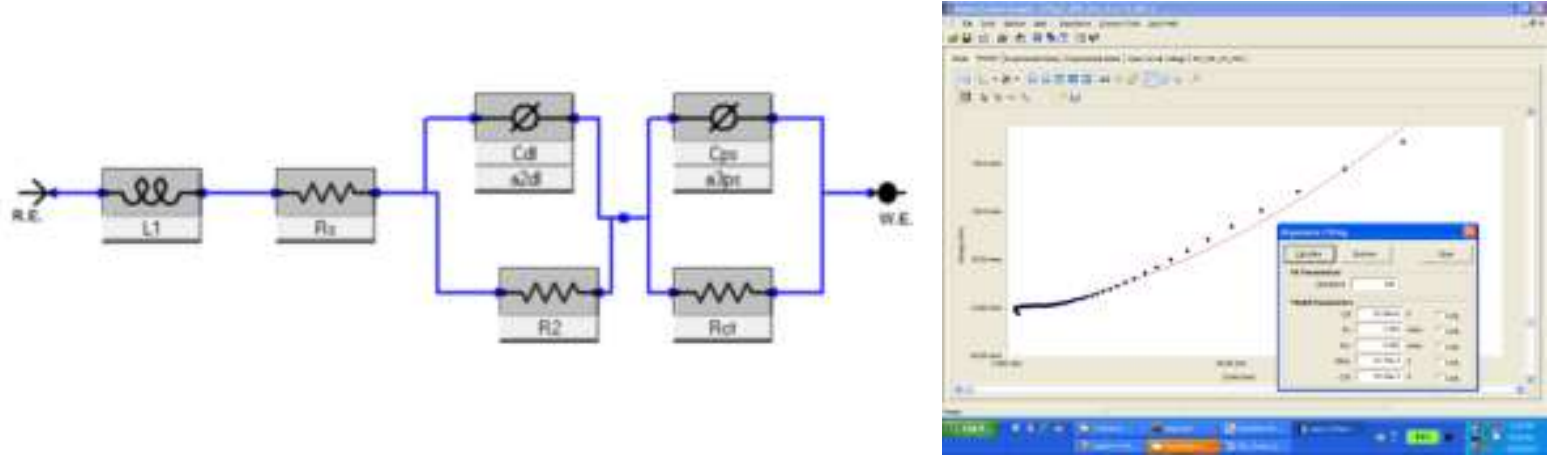

Figure 8: (The electrical circuit model analysis circuit and the corresponding curve fit (Nyquist Plot) 
1) $\mathrm{L} 1$ - Inductance in $(\mathrm{H})$,

3) Cdl - Double Layer Capacitance $\left(S^{*} \mathbf{S}^{\wedge} \mathrm{a}\right)$,

5) R2 - Fiber Mat Resistance (ohms),

7) a3ps ( Fractional Constant ),
2) Rs -Solution and Hardware Resistance (ohms),

4) a2dl (Fraction constant),

6) Cps - Pseudo Capacitance $\left(S^{*} s^{\wedge} a\right)$,

8) Rct - Charge Transfer Resistance (ohm).

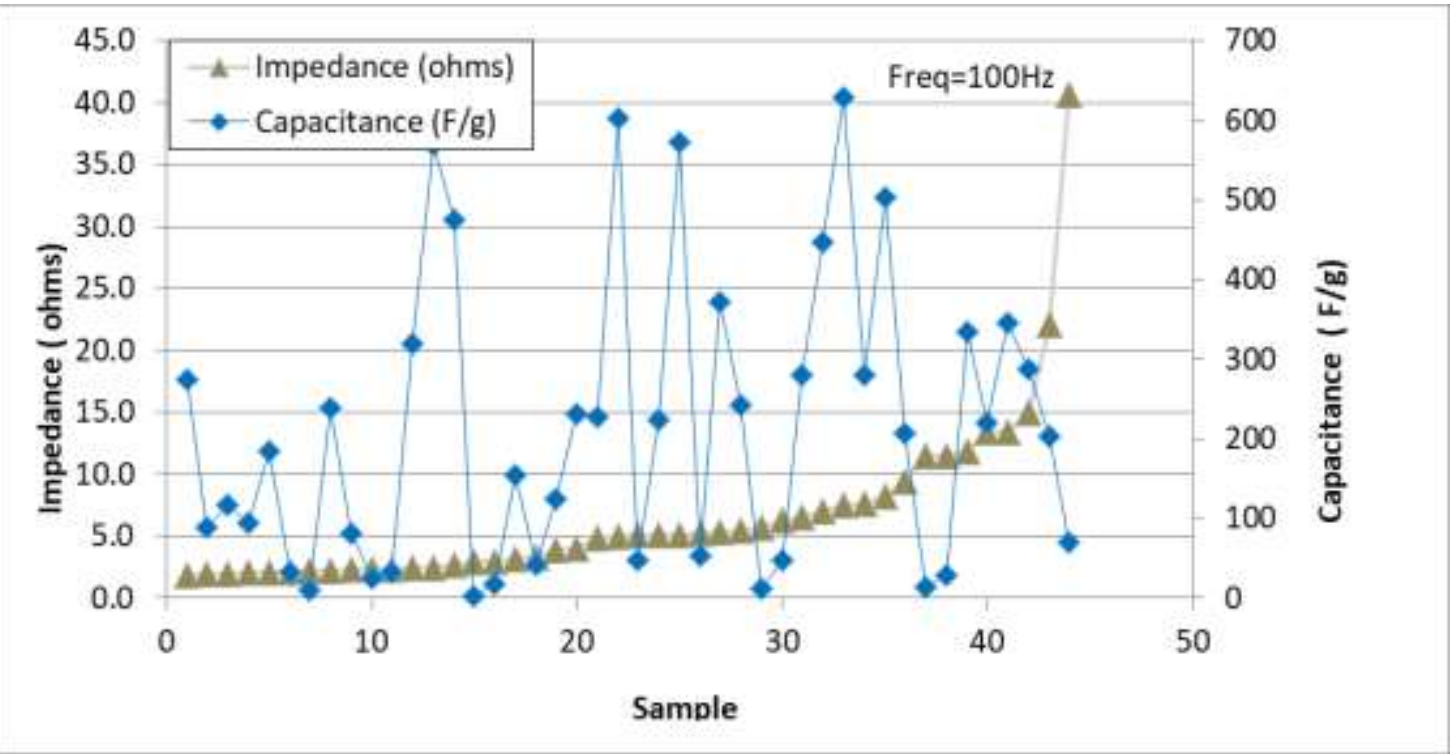

Figure 9: Electrode Nanofiber Impedance (ohms) vs. Capacitance ( F/g ) @ 100Hz Frequency; Top graph sorted with increasing impedance

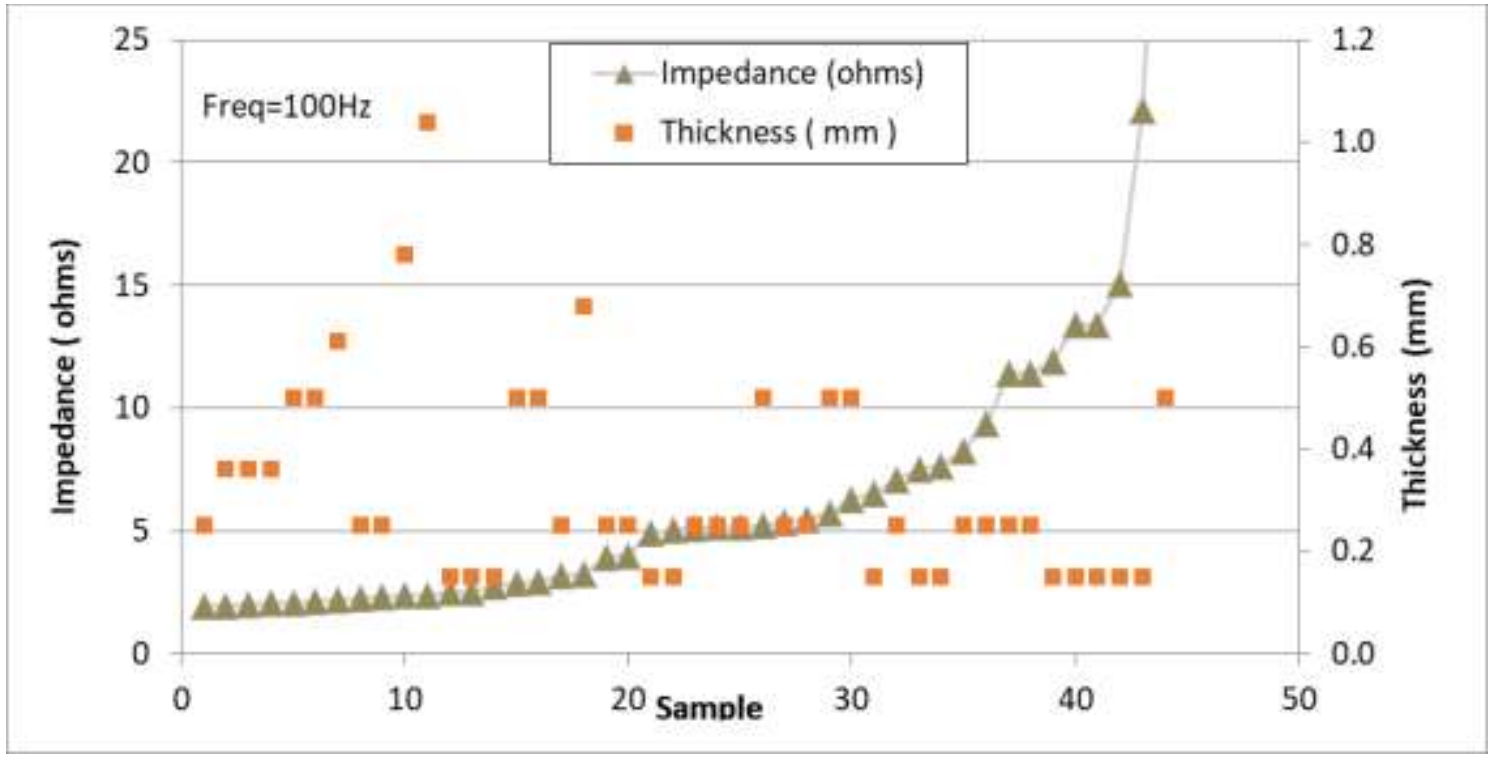

Figure 10: Electrode Nanofiber Impedance (ohms) vs. Thickness (mm) @ 100Hz Frequency

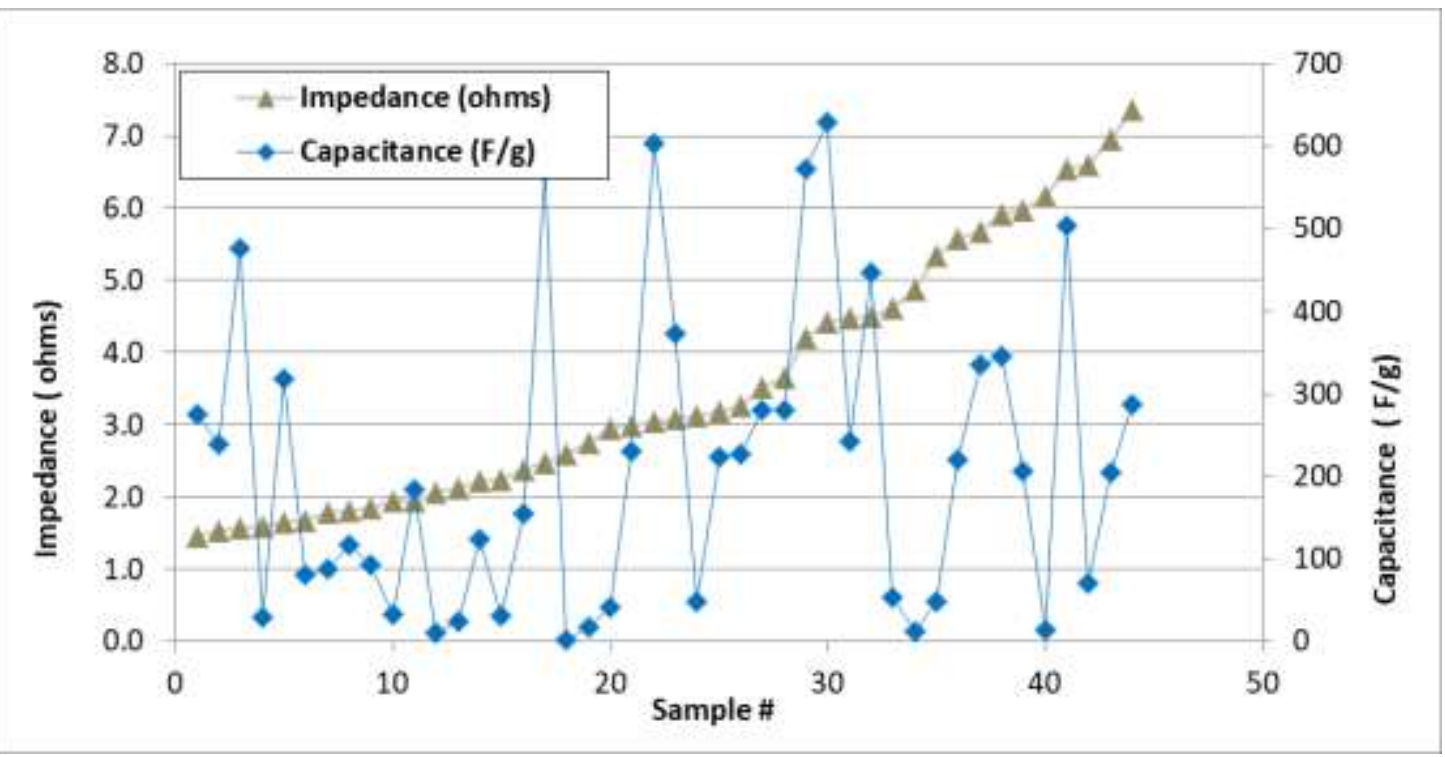




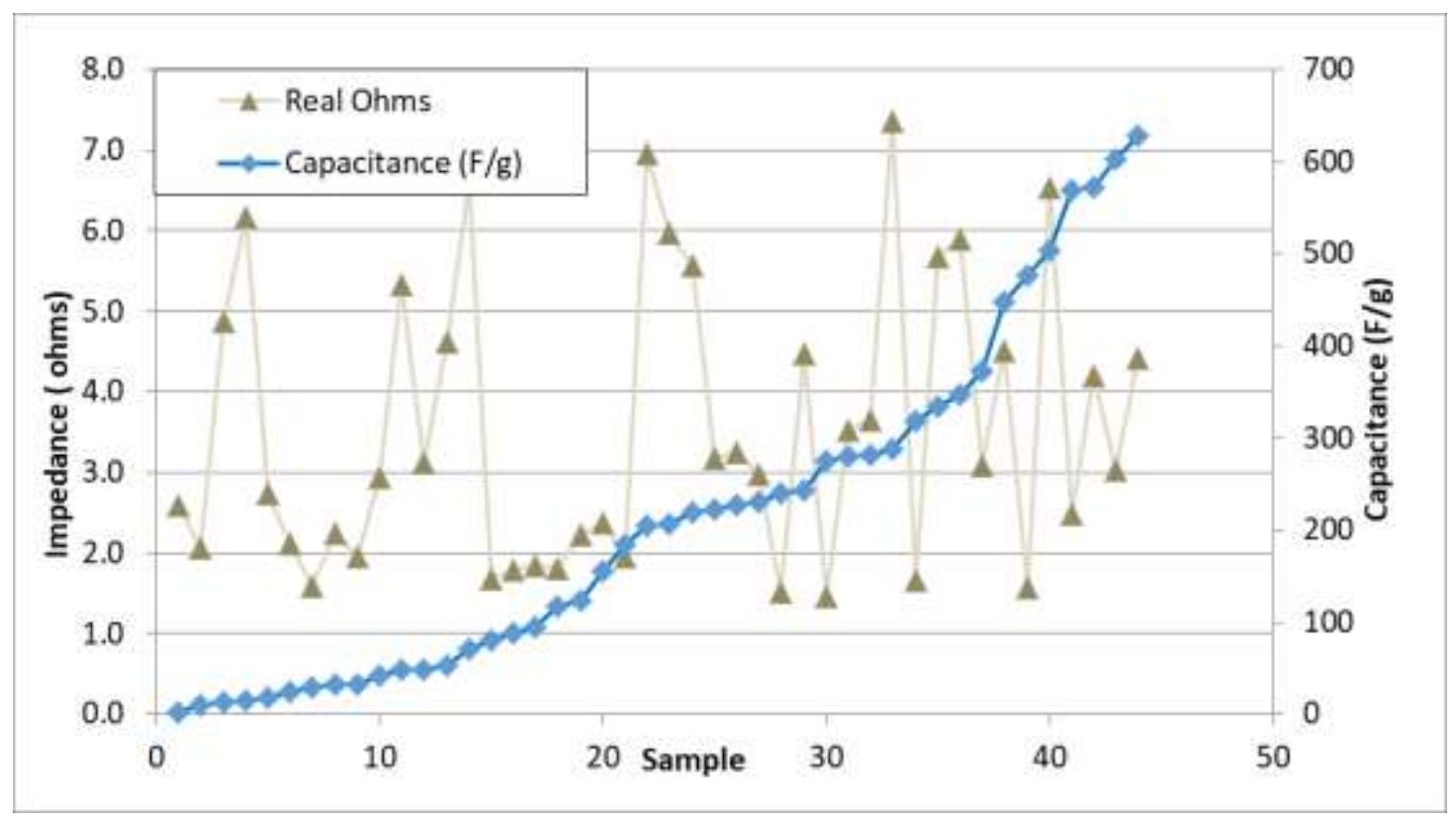

Figure 11: Electrode Nanofiber Capacitance ( F/g ) vs. Impedance (ohms) @ 1kHz Frequency; Top graph sorted with increasing impedance, Bottom graph with increasing capacitance.

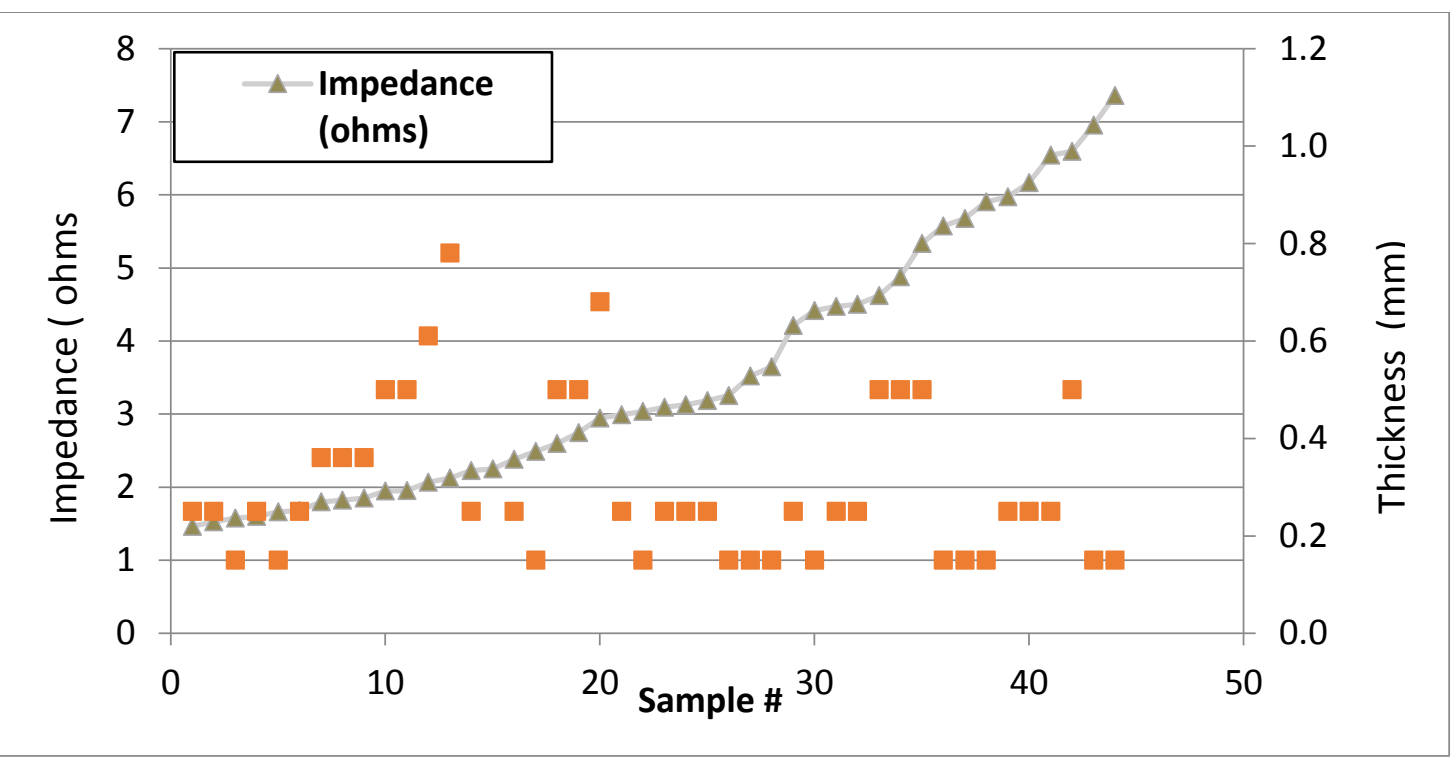

Figure 12: Nanofiber Electrode Thickness (mm) vs. Impedance (ohms) @ 1kHz Frequency

From the tested samples, the overall data indicates no correlation between impedance and capacitance values as seen in Figure 9 at $100 \mathrm{~Hz}$ and Figure 11 at $1000 \mathrm{~Hz}$ Frequency. However, the breakdown of the various samples showed mixed results of; good correlations between lower impedance resulting in higher capacitance; while other samples show higher impedance correlating to both higher and lower capacitance values and finally, samples that showed lower impedance corresponding to lower and higher capacitance values. For example, lower impedance did correlate to overall higher capacitance for the $35 \%$ graphene with 8\% PAN in Figure 31.Also, higher impedance values did correlate to overall higher capacitance in the 8\% PAN only samples in Figure 32. However, Lower impedance values did also correlate to higher and lower capacitance in the $15 \%$ \& $25 \%$ graphene, $10 \%$ (CB) and $8 \%$ PAN samples in Figure 27 \& Figure 28. Furthermore, no correlation between impedance and capacitance was observed in 25\% graphene with $8 \%$ PAN samples in Figure 30 and the 35\% graphene, 10\% (CB) and 8\% PAN Figure 29.

The overall measured impdance is shown in Figure 9. The highest capacitance values were for the $25 \%$ \& $15 \%$ graphene with $8 \%$ PAN and the $8 \%$ PAN-only samples, with capacitance values of 629,569 and $572 \mathrm{~F} / \mathrm{g}$ and an impedance values of 7.5, 2.5 and 5.2 ohms respectively. The impedance range of the capacitors with $100 \mathrm{~F} / \mathrm{g}$ or less is 2.0 to $6.2 \mathrm{ohms}$ with one value of $40.7 \mathrm{ohms}$ with $71 \mathrm{~F} / \mathrm{g}$ value and it's believed to be an outlier Figure 24 . The impedance range of the capacitors between 100 to $300 \mathrm{~F} / \mathrm{g}$ is 1.9 to $15.1 \mathrm{ohms}$ with the highest capacitance of $288 \mathrm{~F} / \mathrm{g}$ with an impedance of $15.1 \mathrm{ohms}$ from the $15 \%$ graphene with $8 \%$ PAN; and the lowest capacitance of this range was $117 \mathrm{~F} / \mathrm{g}$ with an impedance of $1.9 \mathrm{ohms}$ from the $15 \%$ graphene with $8 \%$ PAN and $10 \%$ (CB) Figure 25.

In addition, No correlation was observed between the Impedance value and the thickness of the samples tested as shown in Figure 10 and Figure 12 . However, weak correlation was observed between the carbon mole content of the samples and impedance values, as the average impedance values were shifted higher in the lower carbon mole content samples, which corresponded to the highest capacitance values. And the average impedance values were shifted lower in the higher mole carbon samples which had the lowest capacitance values as shown in Figure 13. Furthermore, the Spectrum and image analysis performed on the nanofibers indicates the lower carbon mole content samples had an amorphous structure, and the higher carbon mole samples had a turbostratic structure Figures 18 and 19. 
From the results above, the conclusion is that mole content and structure have a weak influence on impedance and are not a major factor. This is contrary to Capacitance Energy ( Farads ), where mole content is a major contributor in capacitance energy, with lower carbon mole content corresponding to higher capacitance [1]. This implies that impedance is independent or semi-independent of the energy field or storage. In other words, this provides an alternative control point to control or increase energy, an estimated ( 2-10X times ), that can be retrofitted to existing systems; or increase the energy storage beyond traditional levels by adjusting/controlling impedance. It is peculiar to see that the average impedance values of both the higher carbon mole content samples and the lower carbon mole content samples are about the same and the average value is about $3.0 \mathrm{ohms}$ as seen in Figure 15. From the tested samples, the data is indicating Impedance is varying. The mechanism of varying impedance is unclear. However, it is thought to mimic the energy level of matter (atoms); therefore impedance is a Field, a wrapper that holds or contains the stored energy and expands and contracts accordingly, Impedance is believed to be independent, yet intertwined with the energy Field of matter; more information, that is leading to this prediction, to be provided in next publication (Insha'-ALLAH).

Note, a possible path to impedance stability is ion desolation and nitrogenated functionalities that are thought to cause pseudo-faradaic charge transfer as reported by Ania et al. [18] and Frackowiak et al. [19]. This research is concluding that our conventional understanding of impedance is limited in scope. New approaches and further research is needed to better understand impedance behavior. A better understanding of impedance is essential to a breakthrough in energy storage from capacitors and batteries, to electric generation and distribution, magnetic levitational, medical drugs and other improvements.

\subsection{Spectrum and Image Analysis}

Using a Hitachi Ltd ( S-3000N), Energy Dispersive X-ray ( EDX ) spectroscopy from Oxford Instruments to measure the chemical composition of several samples: The $15 \%$ and $25 \%$ graphene with $10 \%$ (CB) and $8 \%$ PAN; the $15 \%$ graphene with $8 \%$ PAN; and the $8 \%$ PAN only samples were taken. The spectrum analysis indicates that the main composition of the samples is carbon and nitrogen, with smaller traces of oxygen Figure 16.

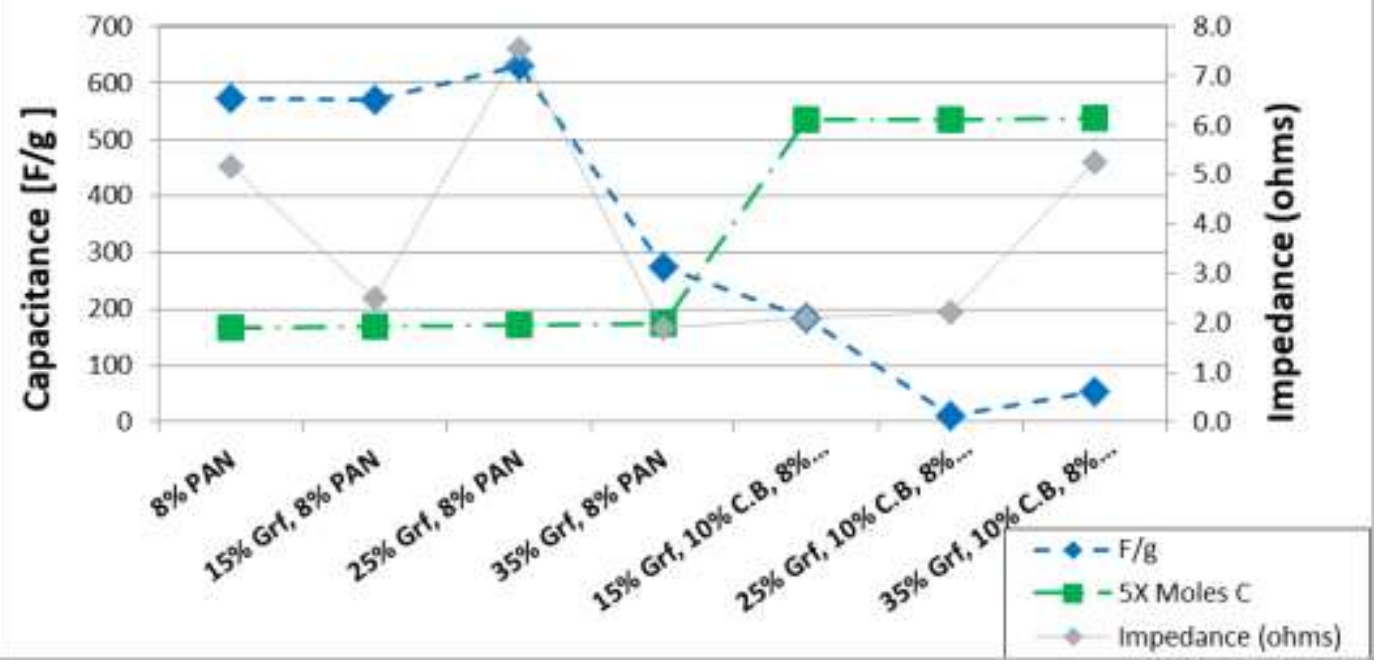

Figure 13: Capacitance (F/g) vs. Impedance (Ohm) \& Moles of Carbon @ 100Hz Frequency

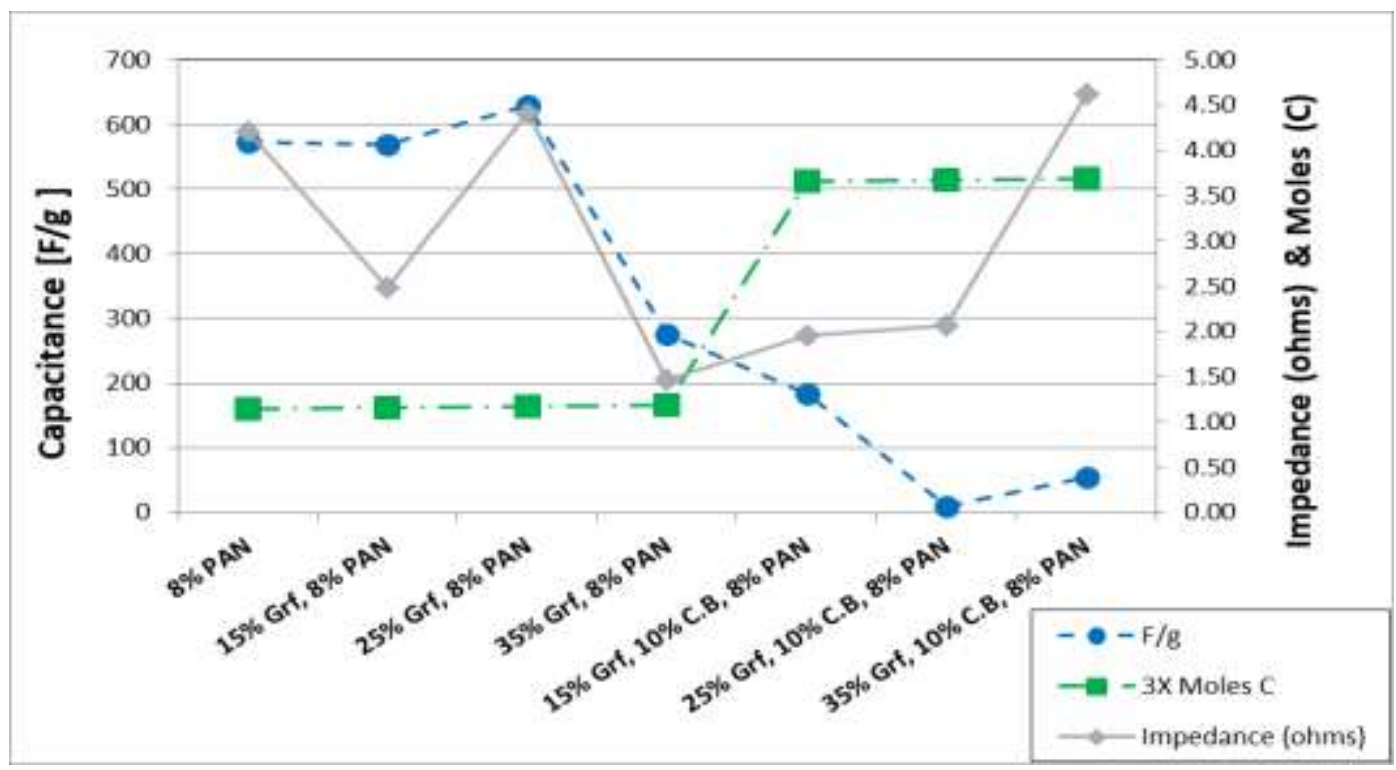

Figure 14: Initial Solution Moles of Carbon vs. Impedance (Ohms) @ 1kHz Frequency 


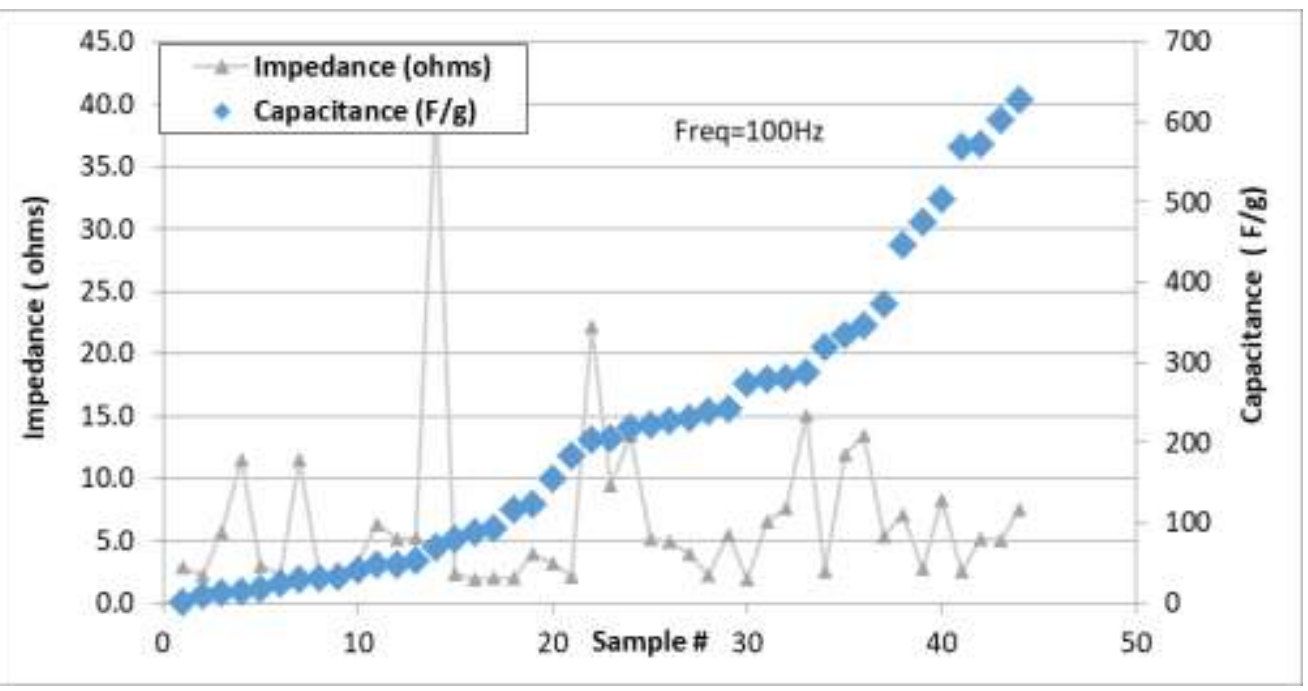

Figure 15: Electrode Nanofiber Impedance (ohms) vs. Capacitance (F/g) @ 100Hz Frequency; Graph sorted with increasing capacitance.
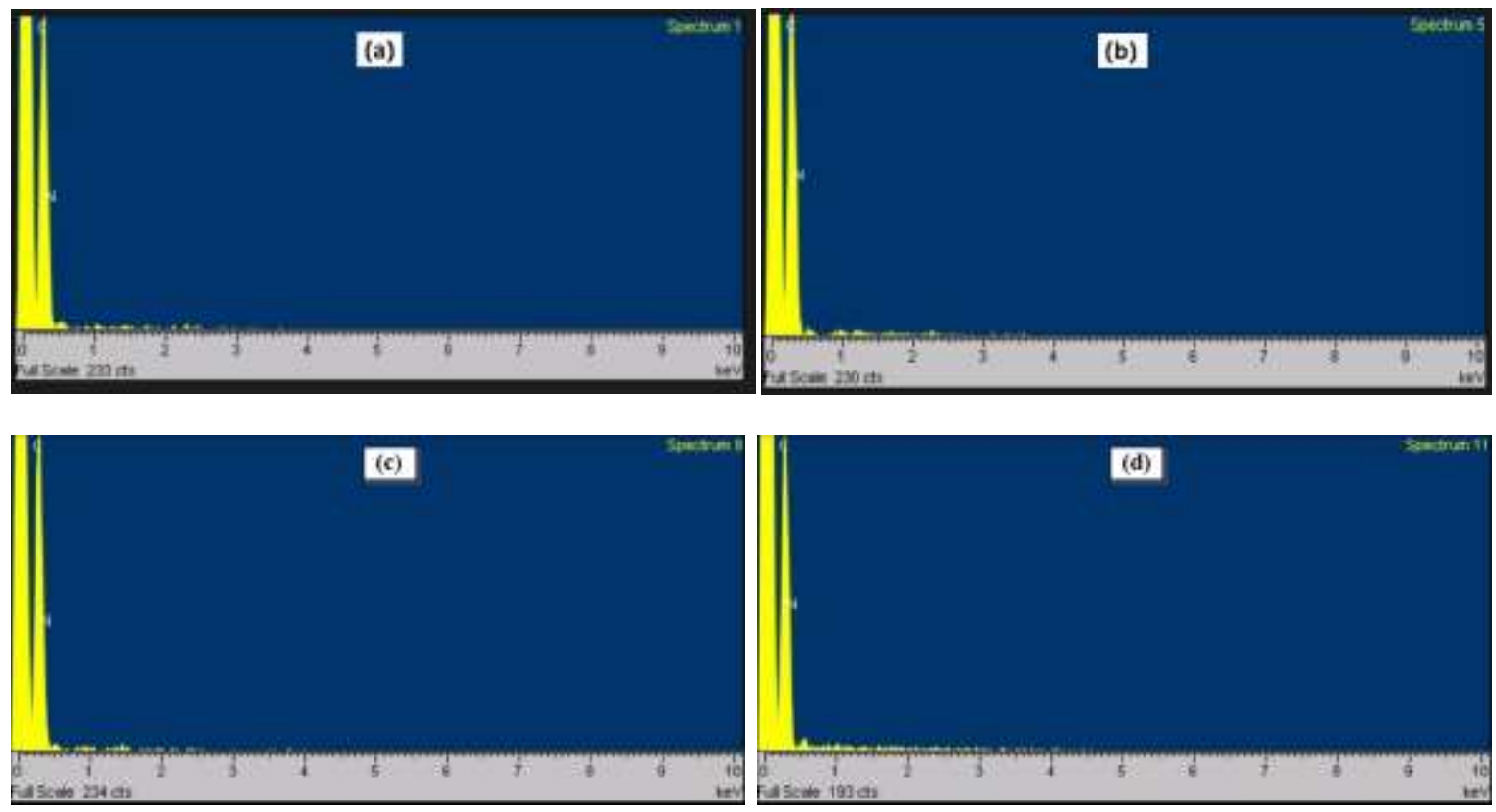

Figure 16: Energy Dispersive X-ray ( EDX) Spectrum analysis

(a) $15 \%$ Graphene, $10 \%$ (CB) with $8 \%$ PAN; (b) 25\% Graphene, 10\% (CB) with 8\% PAN (c) 15\% Graphene with 8\% PAN; (d) $8 \%$ PAN only

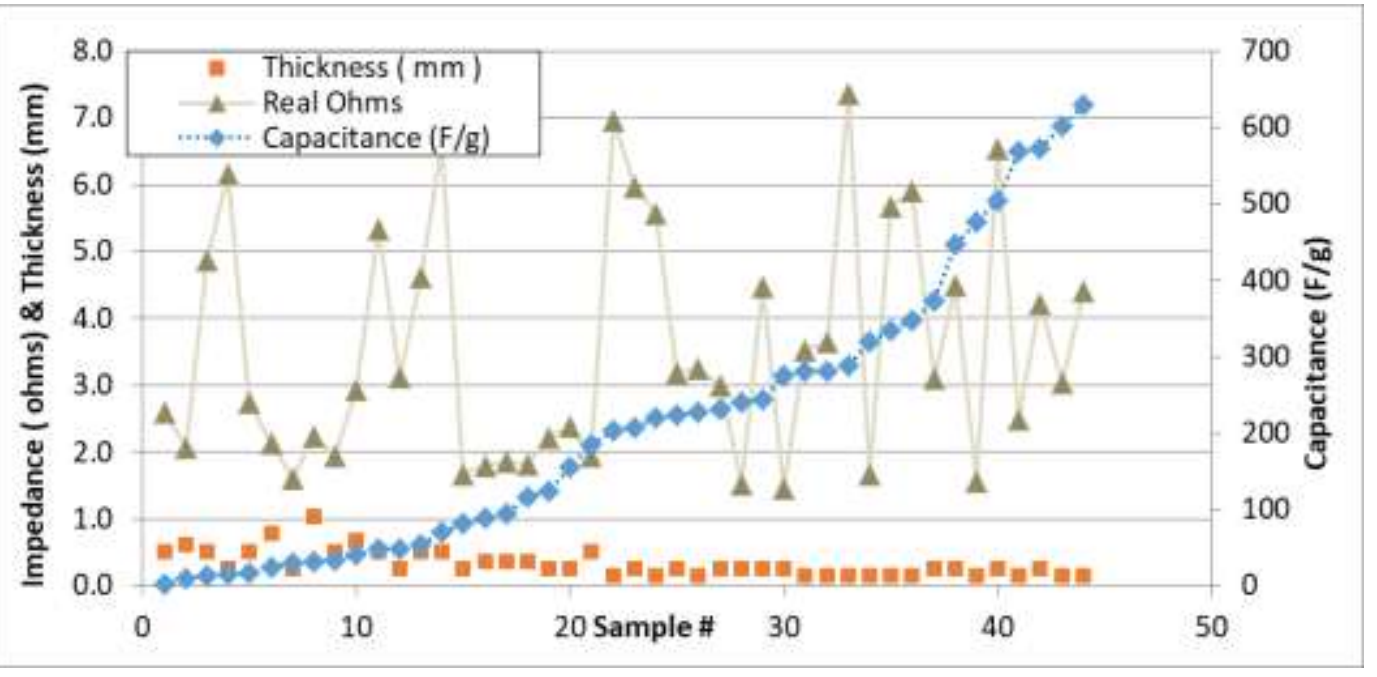

Figure 17: Electrode Nanofiber Impedance (ohms) and mat Thickness (mm) vs. Capacitance ( F/g ) @ 1kHz Frequency.

Transmission electron microscope images were conducted with a JEOL JEM-3010 transmission electron microscope (TEM) with a Gatan digital cameras and Thermo-Noran XEDS. Two samples were imaged and analyzed; a high capacitance value sample of 569 F/g from the $15 \%$ graphene and $8 \%$ PAN with an impedance value of $2.5 \mathrm{ohms}$, and a low capacitance value of $184 \mathrm{~F} / \mathrm{g}$ from the $15 \%$ graphene, $10 \%$ (CB) and $8 \%$ PAN, with an impedance value of 2.1 
ohms. The TEM images are shown in Figures 18 and 19. The TEM image analysis showed an amorphous carbon structure nanofiber mat for the high capacitance value; 15\% graphene and 8\% PAN with an impedance of $2.5 \mathrm{ohms}$ Figure 18. The impedance of $2.5 \mathrm{ohms}$ is in the lower range for the 15\% graphene with 8\% PAN, with an impedance range of ( 2.5 to $15.1 \mathrm{ohms}$ ) and capacitance values of ( 227 to $569 \mathrm{~F} / \mathrm{g}$ ) as seen in Figure 26 . The TEM image analysis of the low capacitance value; $15 \%$ graphene, $10 \%$ (CB) and $8 \%$ PAN; indicates the nanofiber mat is mainly a turbostratic structure Figure 19. The impedance of $2.1 \mathrm{ohms}$ is in the lower range of capacitance for the $15 \%$ graphene, $10 \%$ (CB) and 8\% PAN, with impedance values ranging from (2.0 to 2.1 ohms) and a high impedance value of 40.7 ohms that is believed to be an outlier, and a range of capacitance values of (227 to $569 \mathrm{~F} / \mathrm{g}$ ) as seen in Figure 27 . No correlation between nanofiber mat electrode thickness and Impedance was observed in the tested samples Figure 12.

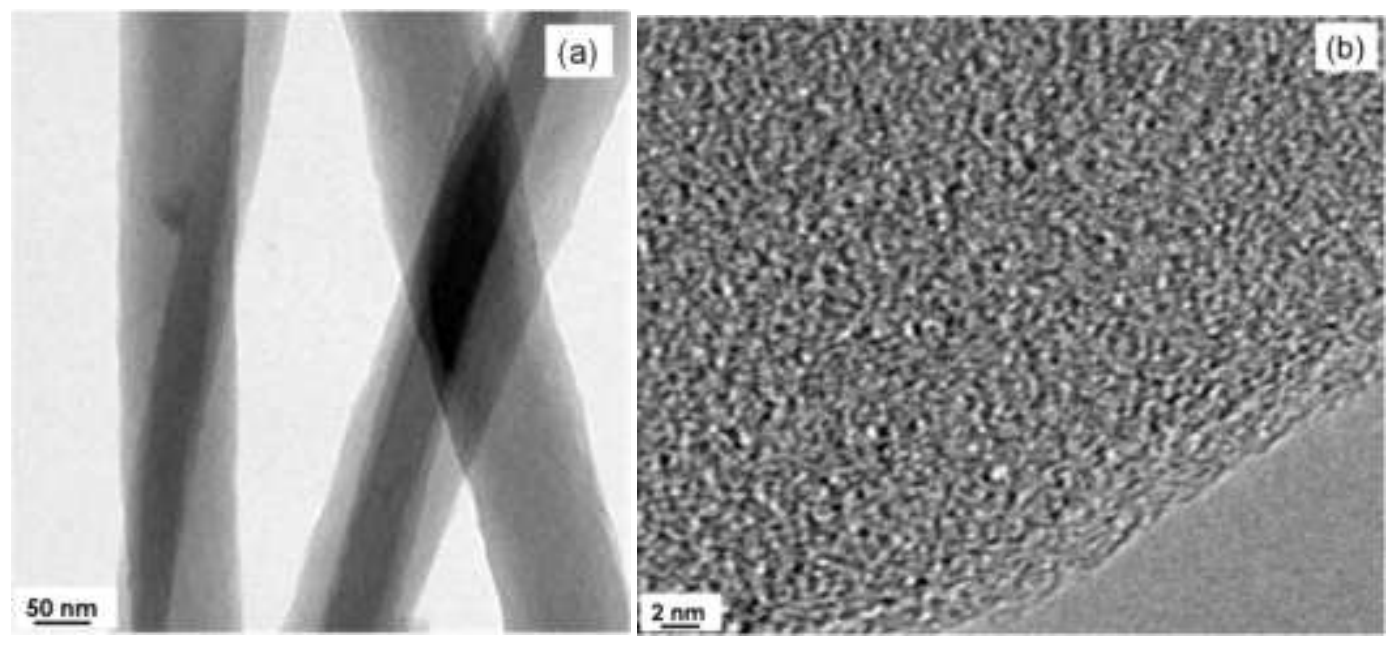

Figure 18: (a)Transmission Electron Microscope (TEM) analysis of 15\% graphene with 8\% PAN nanofiber electrode (b) TEM magnification shows an amorphous carbon (without a crystalline structure), also known as free, reactive carbon and is referred to as allotrope of carbon.

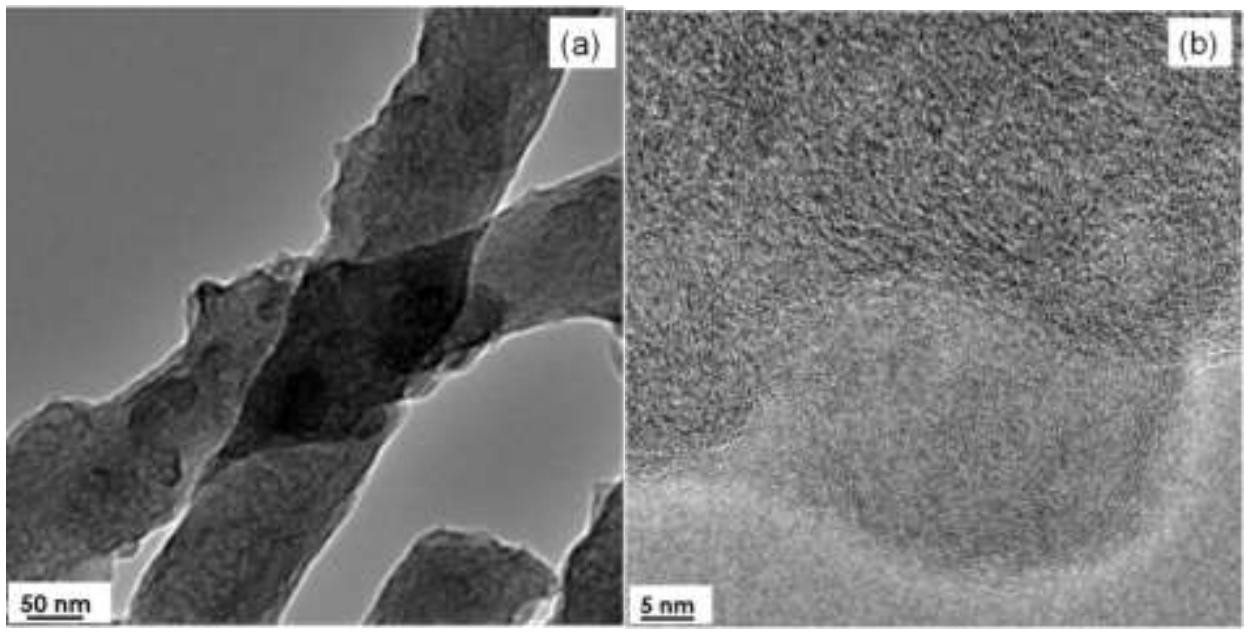

Figure 19: (a) Transmission Electron Microscope (TEM) analysis of 15\% graphene, 10\% (CB) and 8\% PAN nanofiber electrode (b) TEM magnification shows a turbostratic crystallite structure, which is defined as a porous intermediate stage between amorphous carbon and graphene sheet

On the other hand, the TEM image analysis of the lower capacitance 15\% graphene, 10\% (CB) and 8\% PAN suggests that it is mainly a turbostratic structure Figure 19. This structure is a porous intermediate stage between an amorphous carbon and a graphene sheet-type structure.

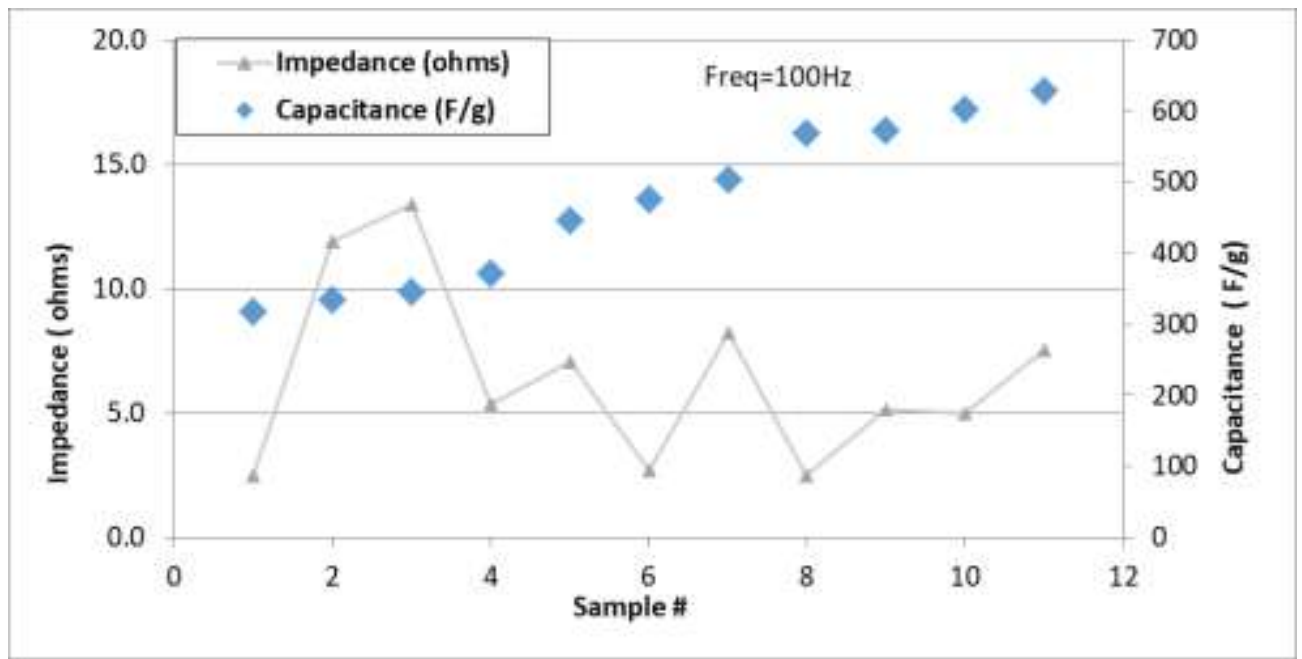


Figure 20: Electrode Nanofiber Capacitance ( F/g ) vs. Impedance ( ohms) \& @ 100Hz Frequency for all samples with capacitance values greater than 300 $\mathrm{F} / \mathrm{g}$

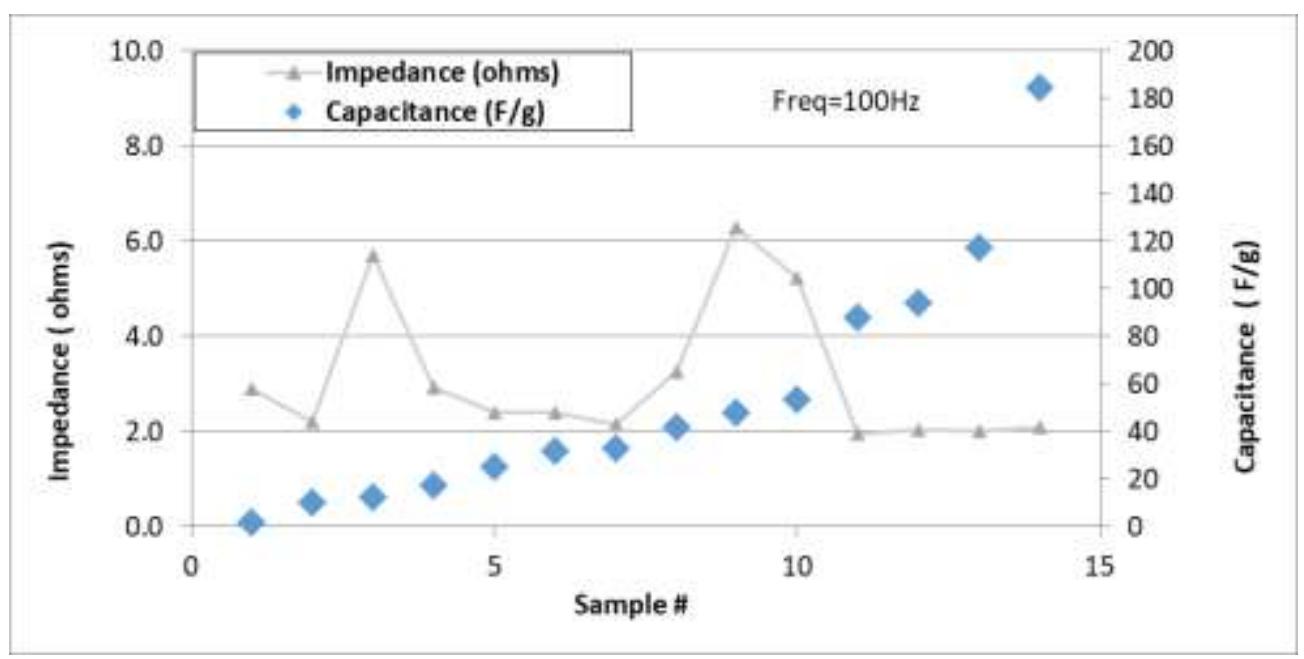

Figure 21: Electrode Nanofiber Impedance (ohms) vs. Capacitance (F/g) @ 100Hz Frequency for the 15\%, 25\% \& 35\% graphene with 8\% PAN and 10\% (CB)

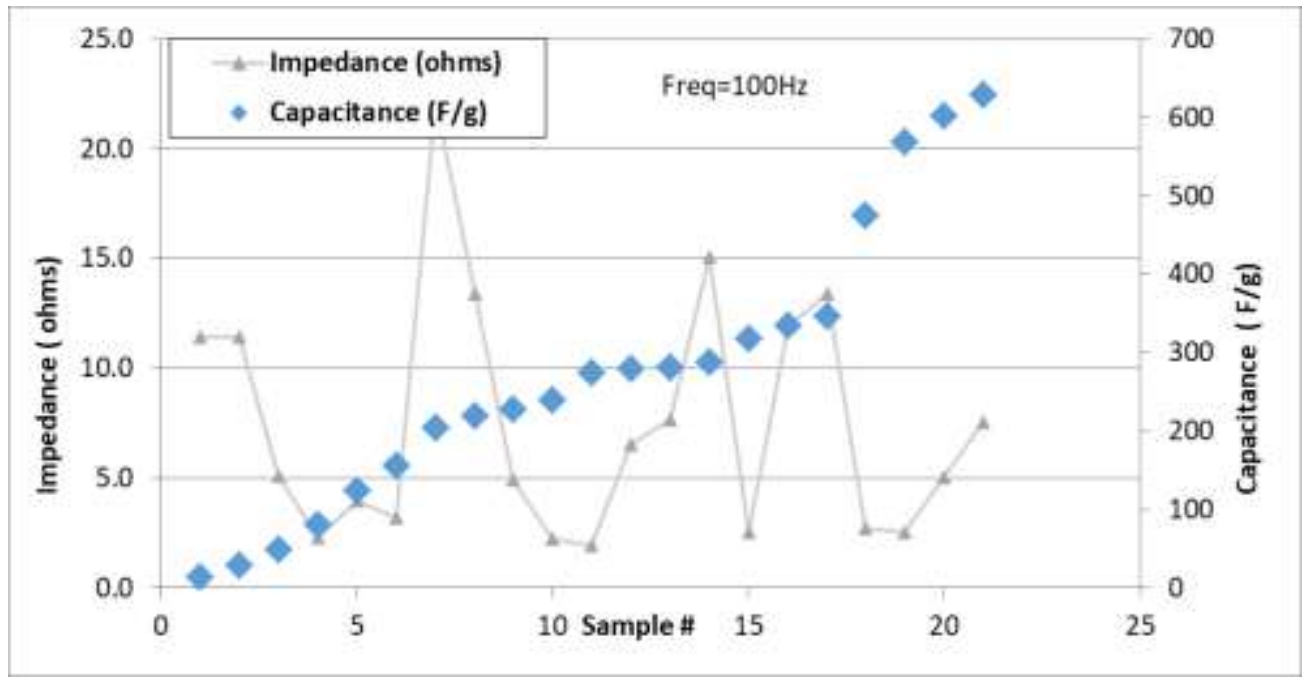

Figure 22: Electrode Nanofiber Capacitance ( F/g ) vs. Impedance ( ohms) \& Thickness (mm) @ 100Hz Frequency for the 15\%, 25\% \& 35\% graphene with $8 \%$ PAN

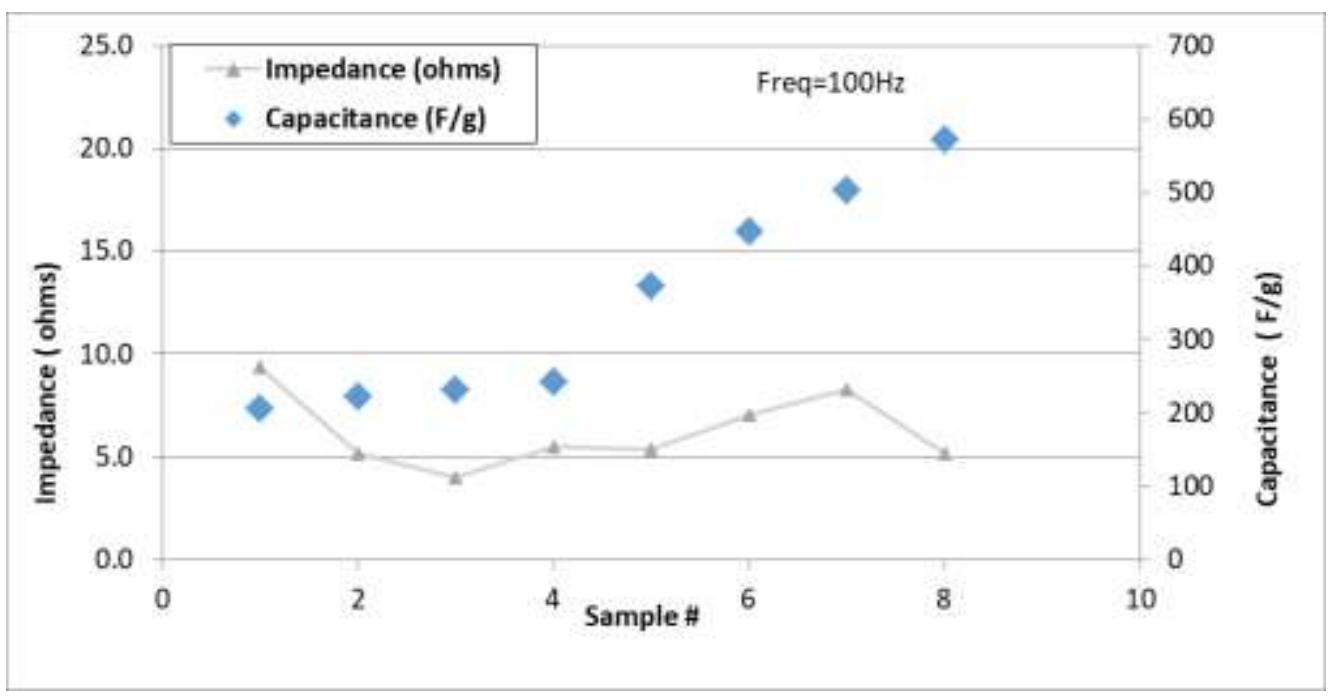

Figure 23: Electrode Nanofiber Capacitance ( F/g ) vs. Impedance ( ohms) \& Thickness (mm) @ 100Hz Frequency for the 8\% PAN-only samples 


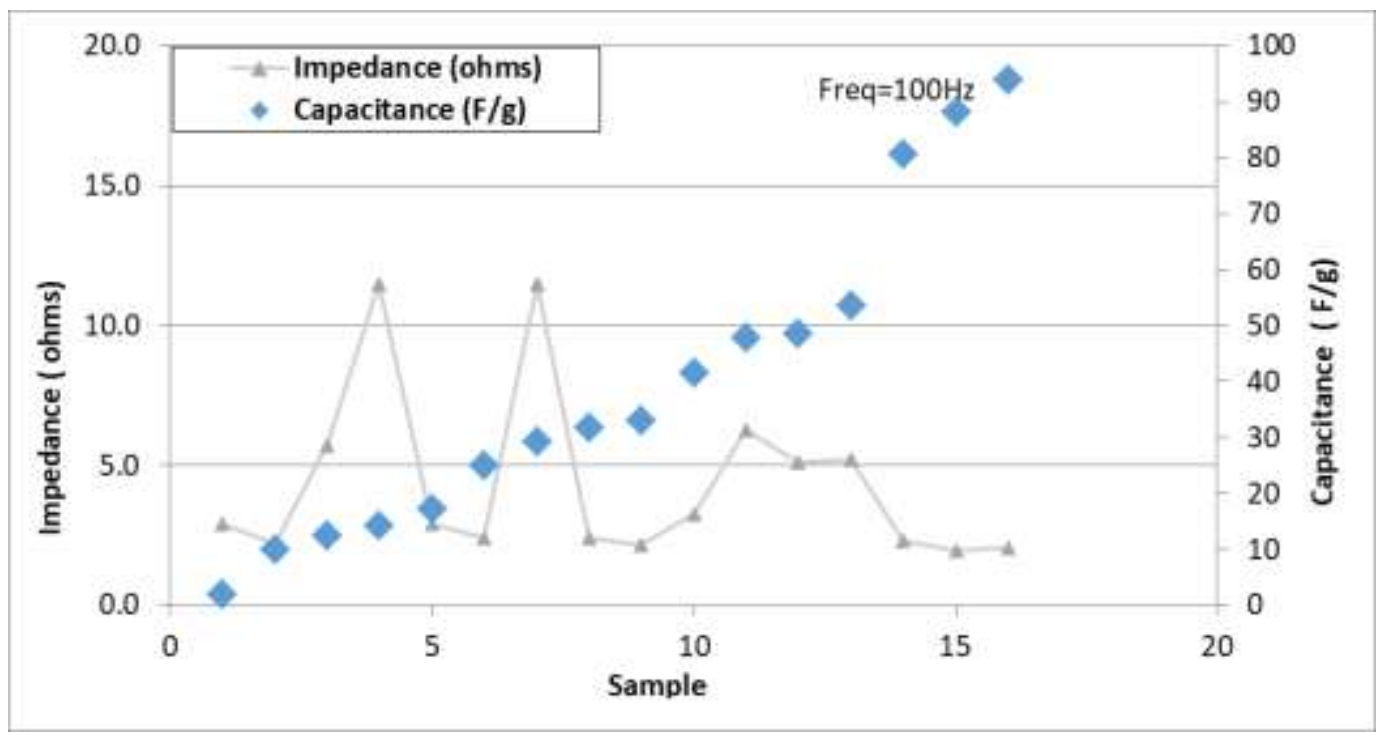

Figure 24: Electrode Nanofiber Capacitance ( F/g ) vs. Impedance ( ohms) \& @ 100Hz Frequency for all samples with capacitance values less than 100 F/g

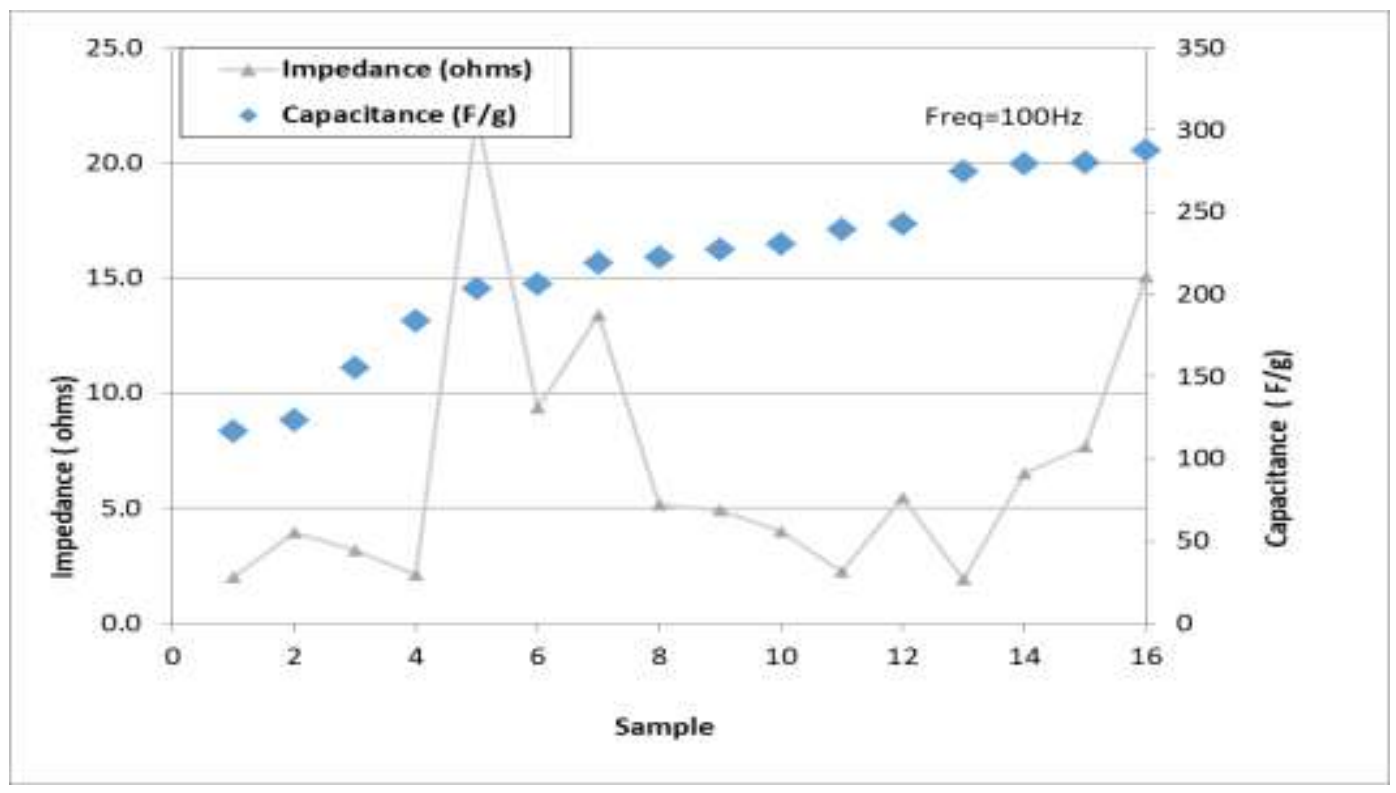

Figure 25: Electrode Nanofiber Capacitance ( F/g ) vs. Impedance ( ohms) \& @ 100Hz Frequency for all samples with capacitance values between 100 to $300 \mathrm{~F} / \mathrm{g}$

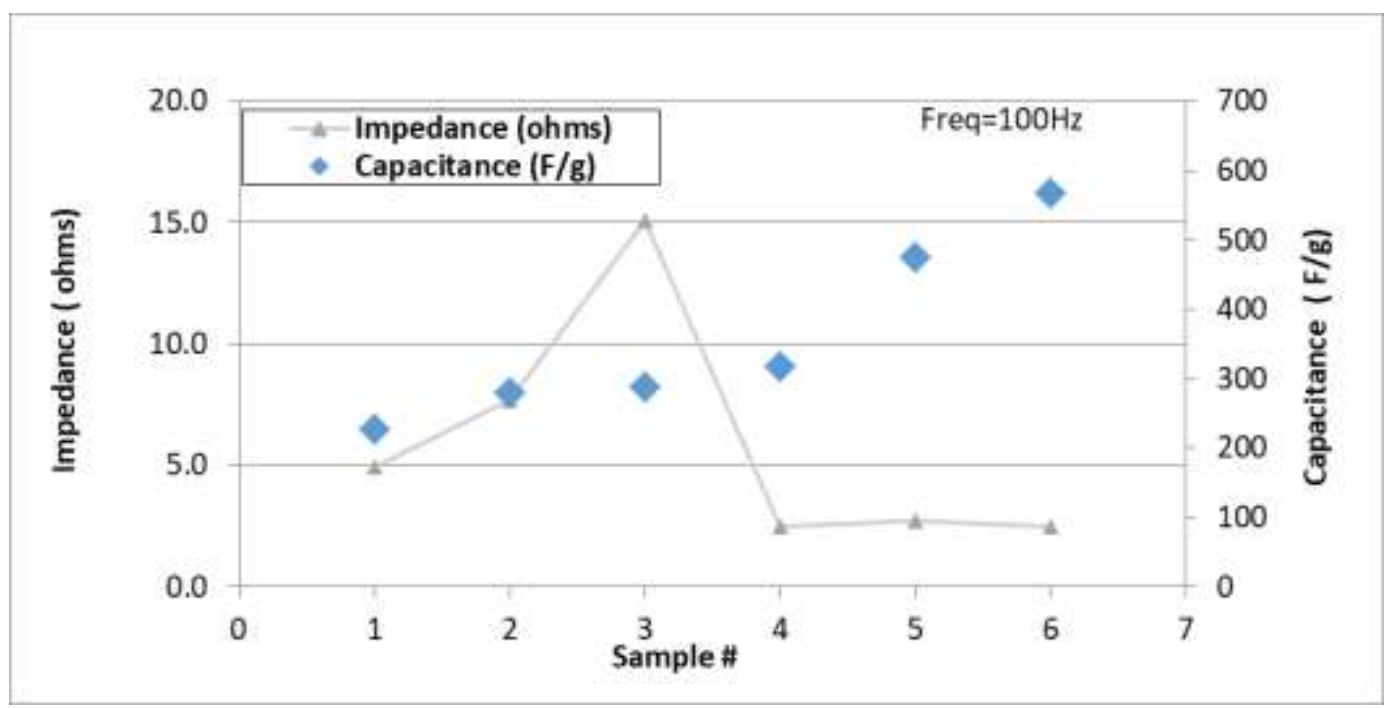

Figure 26: Electrode Nanofiber Capacitance ( F/g) vs. Impedance (ohms) \& @ 100Hz Frequency for the 15\% graphene with 8\% PAN. 


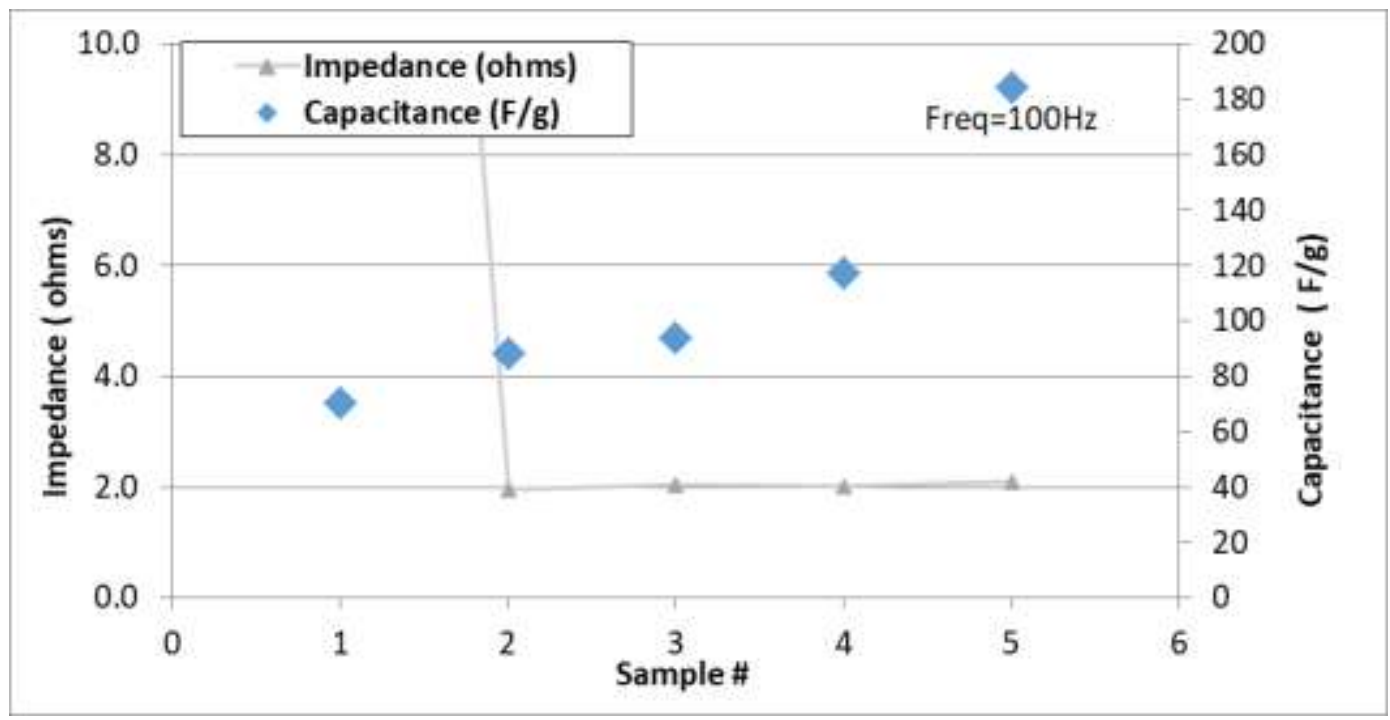

Figure 27: Electrode Nanofiber Capacitance ( F/g ) vs. Impedance ( ohms) \& @ 100Hz Frequency for the 15\% graphene, 10\% (CB) and 8\% PAN .

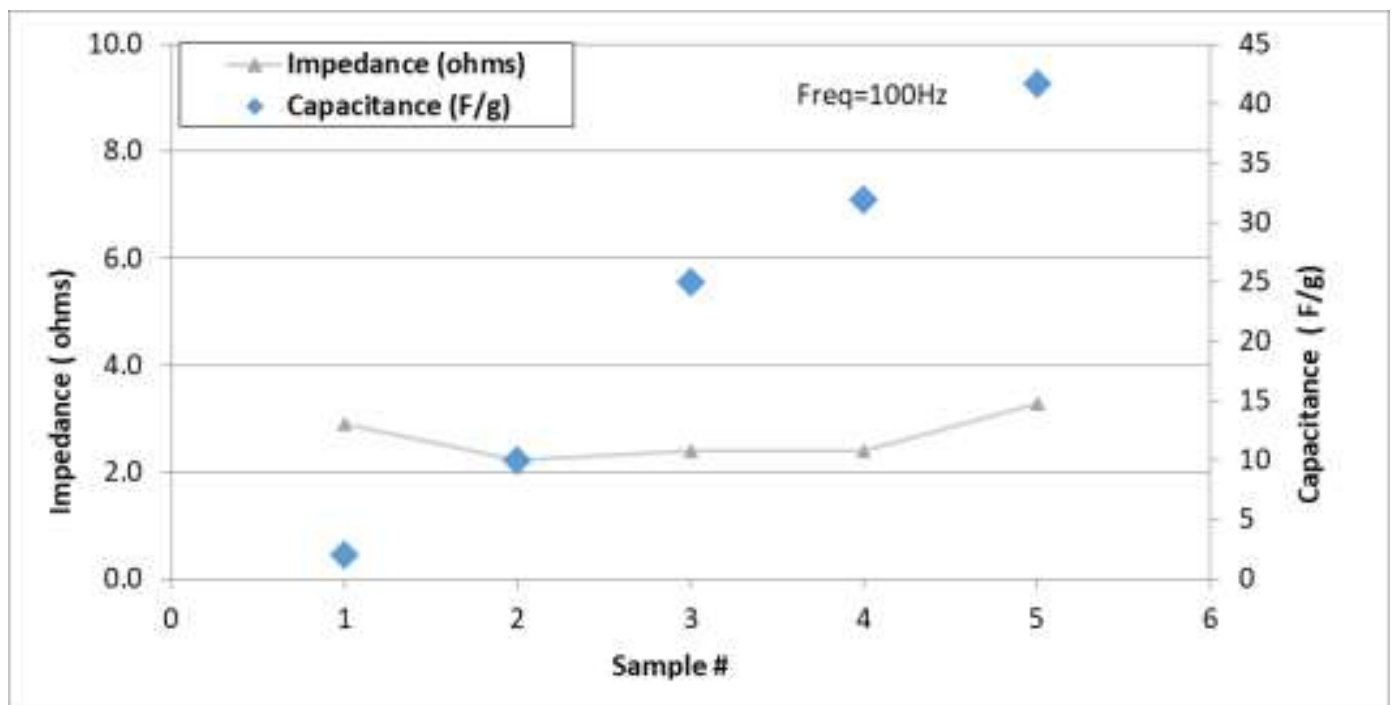

Figure 28: Electrode Nanofiber Capacitance ( F/g ) vs. Impedance ( ohms) \& @ 100Hz Frequency for the 25\% graphene, 10\% (CB) and 8\% PAN .

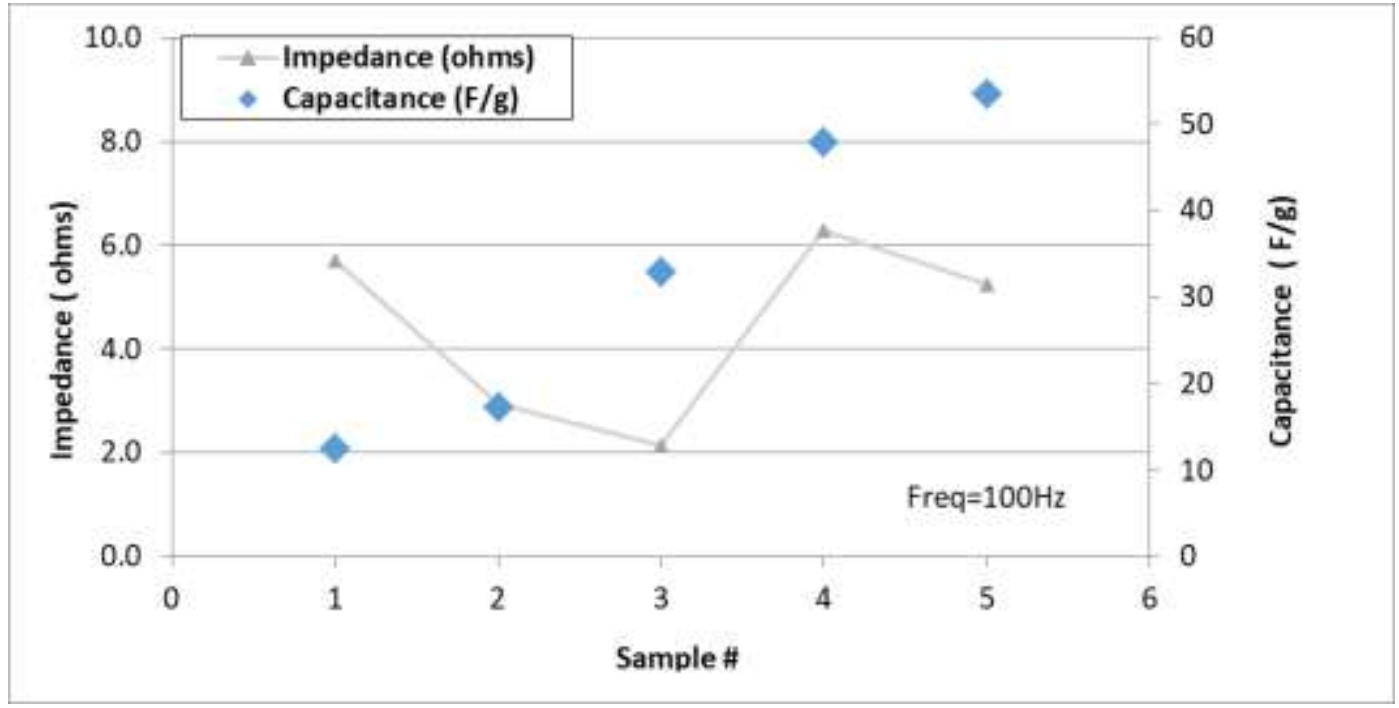

Figure 29: Electrode Nanofiber Capacitance ( F/g ) vs. Impedance ( ohms) \& @ 100Hz Frequency for the 35\% graphene, 10\% (CB) and 8\% PAN . 


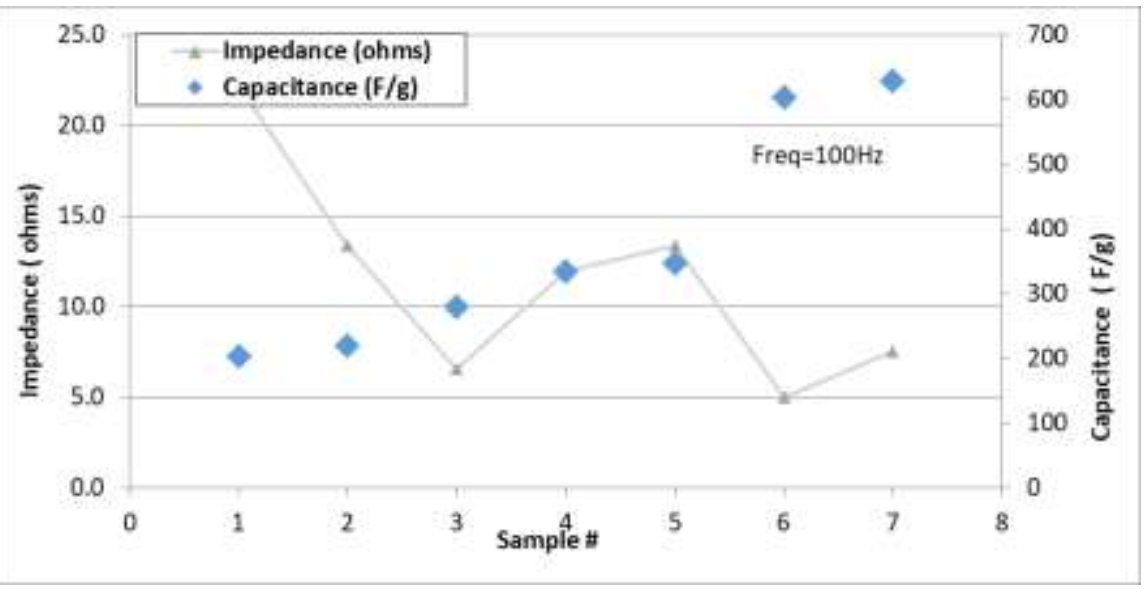

Figure 30: Electrode Nanofiber Capacitance ( F/g ) vs. Impedance ( ohms) \& @ 100Hz Frequency for the 25\% graphene with 8\% PAN.

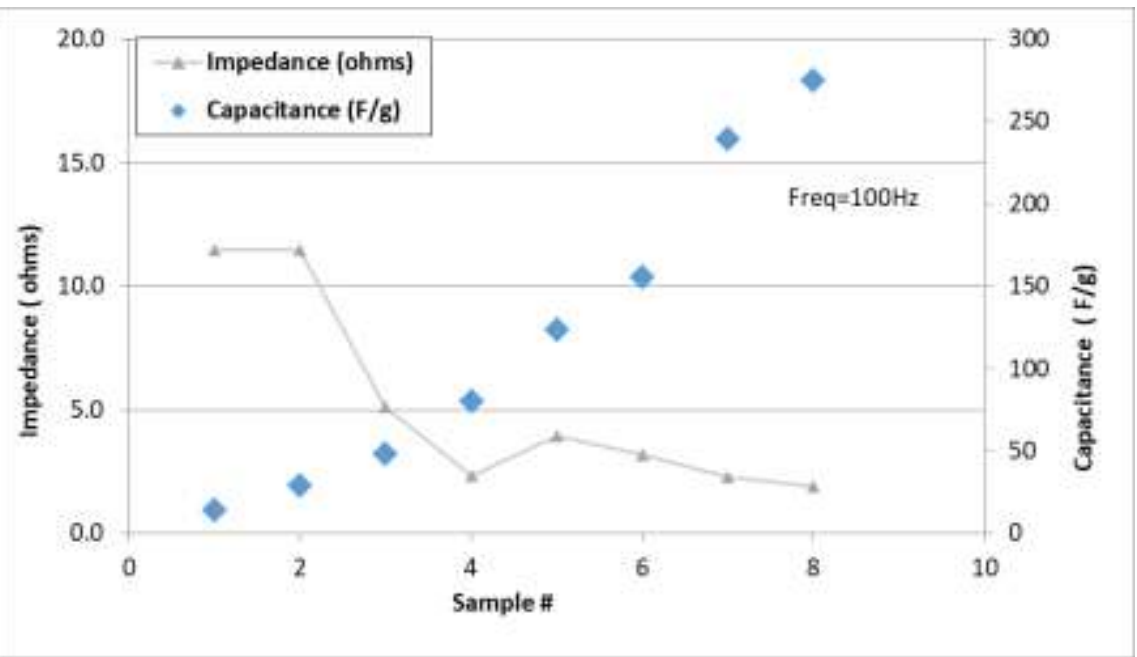

Figure 31: Electrode Nanofiber Capacitance ( F/g ) vs. Impedance ( ohms) \& @ 100Hz Frequency for the 35\% graphene with 8\% PAN.

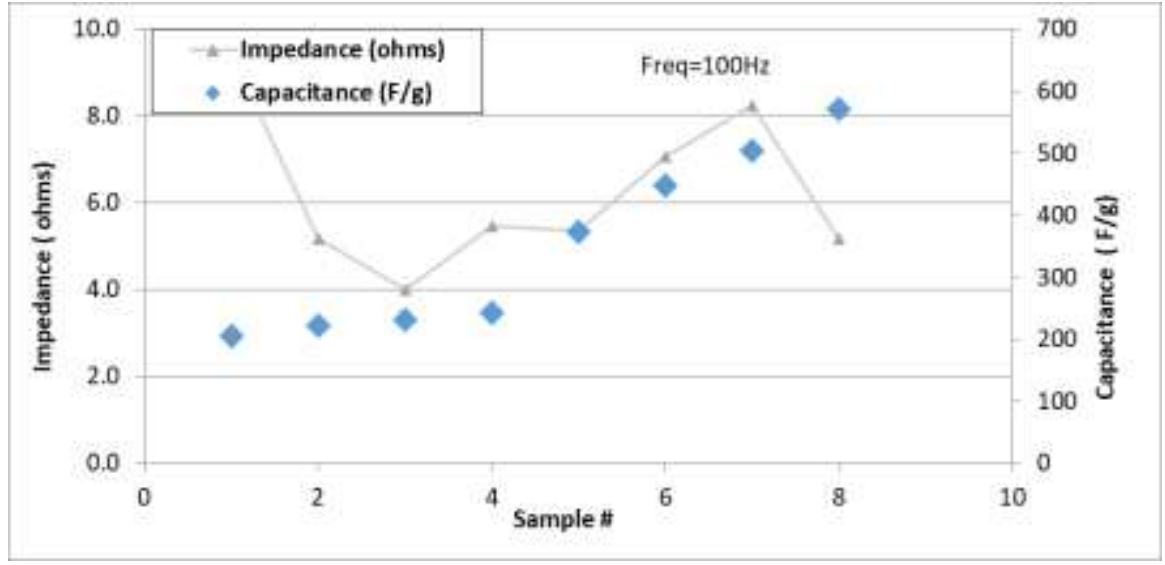

Figure 32: Electrode Nanofiber Capacitance ( F/g ) vs. Impedance ( ohms) \& @ 100Hz Frequency for the 8\% PAN only samples.

\subsection{Conclusion}

The investigation of impedance in supercapacitors with nanofiber electrode mats of varying amounts of graphene and carbon black, formed via the electrospinning process with a base Polyacrylonitrile (PAN) solution, were tested in $1 \mathrm{MH}_{2} \mathrm{SO}_{4}$. A minimum of 5 test samples were constructed of $8 \%$ PAN with, $15 \%, 25 \%$ and $35 \%$ graphene and with/without $10 \%$ carbon black. Also, an $8 \%$ PAN only sample was tested. The (EIS) Impedance plots were initially used to qualitatively compare the impedance values of the tested samples, and capacitance was estimated via circuit model analysis (CMA) of impedance curve fit. However, results of the capacitance using (CMA of EIS data) did not correlate with the experimental capacitance from the (CV) test data. Therefore, a new method of analyzing impedance was developed, that is based on the actual experimental data of (EIS) and (CV). The method utilizes (EIS) real

impedance values, at the frequency that is equivalent to the capacitance discharge time. Therefore, $10 \mathrm{~ms}$ discharge time was used, which corresponds to the impedance value at the $100 \mathrm{~Hz}$ frequency; Which is compared to the experimental capacitance values from (CV) test

The overall data indicates no correlation between impedance and capacitance values. However, the breakdown of the various samples showed mixed results of; good correlations between lower impedance resulting in higher capacitance; while other samples showed higher impedance correlating to higher capacitance, And finally, no correlation of impedance and capacitance, therefore, lower or higher impedance samples corresponding to both lower and 
higher capacitance values. No correlation was observed between Impedance and the thickness of the samples. Furthermore, carbon mole content was not a major factor in determining impedance. Hence, structure is not a major contributor to impedance. This is contrary to Capacitance Energy ( Farads ), where mole content is a major contributor in capacitance energy, with lower carbon mole content corresponding to higher capacitance [1]. This implies that impedance is independent or semi-independent of the energy field or storage. In other words, this provides an alternative control point to controlling or increasing energy an estimated ( $2-10 \mathrm{X}$ times ), that can be retrofitted to existing systems; or increasing the energy storage beyond traditional limits by adjusting/ controlling impedance.

Further research is needed to draw a definite conclusion, however, from the tested samples, the data is indicating that impedance is not constant and is varying. The mechanism of varying impedance is unclear and requires further research. However, it is thought to mimic the energy level of matter (atoms). Therefore, impedance varies or oscillates accordingly to achieve a stable orbit level that is thought, to be independent and intertwined with the energy level of matter. The oscillating or wave behavior type, to achieving impedance level stability, is termed "Impedance or Orbital Level Stability". This research is concluding that our conventional understanding of impedance is limited in scope. New approaches and further research is needed to better understand impedance behavior, which is essential to a breakthrough in energy storage, production and distribution.

\subsection{Acknowledgements}

The author acknowledges and appreciates the use of lab equipment from the University of Illinois at Chicago, USA. This research was self-funded.

\subsection{References}

[1] M.H. Mustafa, A. Zdunek, "Supercapacitor Nanofiber Electrodes Graphene-Based”, Int. J. Electrochem. Sci., Vol. 12, Pp. 2917-2932, 2017.

[2] W. Liu, X. Yam, J. Lang, C. Peng, Q. Xue, "Flexible and conductive nanocomposite electrode based on graphene sheets and cotton cloth for supercapacitors", J. Mater. Chem., Vol. 22, Pp. 17245-17253, 2012.

[3] E. Garcia-Breijo, G. Prats-Boluda, R. Lidon, J. Vicente, Y. Ye-Lin, J. Garcia-Casado, "A comparative analysis of printing techniques by using an active concentric ring electrode for bioelectrical recording", Microelectronics International, Vol. 32, Pp. 107-103, 2015.

[4] K. Kim, “High Operating Voltage Supercapacitor Using PPy/AC Composite Electrode Based on Simple Dipping Method”, J. Chemistry, 314893, Pp. 1-7.

[5] K. Nam, K. Kim, "Manganese oxide film electrodes prepared by electrostatic spray deposition for electrochemical capacitors", J. Electrochem. Soc., Vol. 153, Pp. A81-A88.

[6] S. Al-Hallaj, K. Kiszynski, “Hybrid Hydrogen Systems”, Stationery and Transportation Applications, Pp. 55-94, 2011.

[7] E. Askari, S. Nagbih, "A novel approach to facile synthesis and biosensing of the protein-regulated graphene", Int. J. Electrochem. Sci., Vol. 13, Pp. 886-897, 2018.

[8] Y. Shao, M. El-Kady, L.Wang, Q. Zhang, Y. Li, H. Wang, M. Mousaviae, R. Kaner, “Graphene-based materials for flexible supercapacitors”, Chem. Soc. Rev., Vol. 44, Pp. 3639-3665, 2015.

[9] X. Shi, W. Zhou, D. Ma, Q. Ma, D. Bridges, Y. Ma, A. Hu, "Electrospinning of nanofibers and their applications for energy devices", J. Nanomaterials, 140716, 2015.

[10] X. Mao, T. Hatton, G. Rutledge, "A Review of Electrospun Carbon Fibers as Electrode Materials for Energy Storage", Current Organic Chemistry, Vol. 17, Pp. 1390-1401, 2013.

[11] K. Chan, "Electrochemical characterization of electrospun activated carbon nanofibers as anelectrode in supercapacitors", J. Power Sources, 142, Pp. 382-388, 2005.

[12] C. Kim, Y. Choi, W. Lee, K. Yang, "Supecapacitor performances of activated carbon fiber webs prepared by electrospinning of pMDA-ODA poly (amic acid) solutions", PBFC, Vol. 50, Pp. 883-887, 2004.

[13] D. Wu, Z. Xiao, L. Deng, Y. Sun, Q. Tan, L. Dong, S. Huang, R. Zhu, Y. Liu, W. Zheng, Y. Zhao, L. Wang, D. Sun, "Enhanced Deposition Uniformity via an Auxiliary Electrode in Massive Electrospinning", Nanomaterials, Vol. 135, Pp. 1-12, 2016.

[14] R. Lin, P.L. Taberna, J. Chmiola, D. Guay, Y. Gogotsi, P. Simon, "Microelectrode study of pore size, ion Size, and solvent effects on the charge/discharge behavior of microporous carbons for electrical double-layer capacitors", J. Electrochem. Soc., Vol. 156, Pp. A7-A12, 2009.

[15] M. Toupin, D. Belanger, I. Hill, D. Quinn, “Performance of experimental carbon blacks in aqueoussupercapacitors”, J. Power Sources, Vol. 140, Pp. 203210, 2005.

[16] J. Chmiola, G. Yushin, Y. Gogotsi, C. Portet, P. Simon, P.L. Taberna, “Anomalous increase in carbon capacitance at pore sizes less than 1 nanometer", Science, Vol. 313, Pp. 1760-1763, 2006.

[17] C. Largeot, C. Portet, J. Chmiola, P.L. Taberna, Y. Gogotsi, P.J Simon, "Relation between the Ion Size and Pore Size for an Electric Double-Layer Capacitor", Am. Chem. Soc., Vol. 130, Pp. 2730-2731, 2008.

[18] C. Ania, J Pernak, F. Stefaniak, E. Raymundo-Pĩnero, F. B'eguin, "Polarization-induced distortion of ions in the pores of carbon electrodes for electrochemical capacitors", Carbon, Vol. 47, Pp. 3158-3166, 2009.

[19] E. Frackowiak, Q. Abbas, F. B'eguin, “Review Carbon/carbon supercapacitors”, Journal of Energy Chemistry, Vol. 22, Pp. 226-240, 2013.

[20] F. Ko, Y. Gogotsi, A. Ali, N. Naguib, H. Ye, G. Yang, C. Li, P. Willis, “Electrospinning of continuous carbon nanotube-filled nanofiber yarns”, Adv Mat, Vol. 15, Pp. 1161-1165, 2003.

[21] J. Wang, S. Kaskel, “KOH activation of carbon-based materials for energy storage”, J. Mater. Chem., Vol. 22, Pp. 23710, 2012.

[22] W.G. Pell, B.E. Conway, "Voltammetry at a de Levie brush electrode as a model for electrochemical supercapacitor behaviour”, Journal of Electroanalytical Chemistry, Vol. 500, Pp. 121-133, 2001. 